Panorama ECONómico, Volumen IV, No. 8, enero-junio, 2009, pp. 101-148

\title{
EFECTOS DE LAS CRISIS ANTICIPADAS Y NO ANTICIPADAS SOBRE EL CONTAGIO FINANCIERO INTERNACIONAL
}

\author{
Mario Lagunes* \\ Karen Watkins**
}

\begin{abstract}
RESUMEN
La literatura sobre contagio financiero internacional señala que crisis financieras anticipadas ocasionan un efecto contagio nulo; lo contrario ocurre con crisis no anticipadas. Este artículo propone una metodología para comparar el contagio que pudo haber ocasionado la crisis de Hong Kong en 1997 (no anticipada) y la argentina en 2001 (anticipada), sobre Brasil y México. Como variables de contagio se toman los rendimientos accionarios y las tasas de interés reales. Los resultados muestran que la anticipación de la crisis argentina evitó el contagio sobre México y Brasil; por el contrario, la crisis en Hong Kong sí contagió a estos mercados.

Palabras clave: Crisis financieras, contagio, anticipación de crisis, mercados bursátiles, tasas de interés

Clasificación JEL: G01, G15

* Universidad Popular Autónoma del Estado de Puebla, Tel: +52 222 2299400. Correo electrónico: <marioalberto.lagunes@upaep.mx>.

** Universidad Popular Autónoma del Estado de Puebla, Facultad de Economía y Centro de Investigación e Inteligencia Económica. Benemérita Universidad Autónoma de Puebla, Facultad de Economía. Miembro del Sistema Nacional de Investigadores (SNI C). Correo electrónico: $<$ karen.watkins.f@gmail.com>.

Agradecemos a María Josefina Rivero por su apoyo en la revisión de estilo del artículo, así como a Mariano Rojas y Maikol Elizondo por sus valiosas sugerencias.
\end{abstract}




\begin{abstract}
The literature on international financial contagion explains that anticipated crises do not produce contagion; the contrary occurs with non-anticipated ones. This paper proposes a methodology to compare the contagion effects that could have occurred with the Hong Kong-1997 crisis (non-anticipated) and the Argentinean one (anticipated), over Brazil and Mexico. As contagion variables we use stock returns and real interest rates. Results show that the anticipation of the Argentinean crisis avoided the contagion effects over Mexico and Brazil; on the contrary, the Hong Kong crisis had contagion outcomes over these markets.

Keywords: Financial crises, contagion, anticipation of crises, stock markets, interest rates

JEL classification: G01, G15
\end{abstract}

\title{
1. INTRODUCCIÓN
}

Durante la década de los noventa, ocurrieron en varias regiones del mundo eventos donde los movimientos adversos en las variables económicas y financieras de un país afectaban las mismas variables de otros países; esto se observaba de manera más evidente en el comportamiento que registraban las bolsas de valores.

Conforme esta situación se iba repitiendo, surgió en la literatura financiera un gran número de investigaciones tratando de explicar el fenómeno. A éste se le llegó a denominar contagio financiero o simplemente contagio (Karolyi, 2003; Dungey et al., 2005).

A pesar de haberse realizado un número importante de estudios, algunos autores como Forbes y Rigobon (2002) y Boschi (2005) llegaron a la conclusión de que no existía ningún consenso acerca de lo que es el contagio financiero y de sus características.

Sin embargo, conforme estos episodios comenzaron a ser menos frecuentes a partir de 1999, se llega a la conclusión de que los eventos de contagio 
financiero tienden a disminuir, sin que eso implique que no puedan ocurrir nuevamente en el futuro (Didier et al., 2006).

Si bien han transcurrido ya casi once años desde el último período de contagio significativo ocasionado por la crisis rusa de 1998, el ciclo económico sigue siendo vigente y no hay garantía de que no haya otra crisis en algún mercado económico y financieramente importante; incluso esto puede darse sin previo aviso, lo que podría originar otro episodio de contagio financiero.

Efectivamente, el 27 de febrero de 2007, la Bolsa de Valores de Shangai presentó una caída del $8.8 \%$ lo que revivió entre los inversionistas los temores de la ocurrencia de una nueva crisis asiática similar a la ocurrida en 1997.

Ante este evento, todas las bolsas del mundo tuvieron caídas importantes. El índice Dow Jones tuvo un retroceso de más de 400 puntos, el mayor desde el 11 de septiembre de 2001 (Reuters, 2007). Para el 5 de marzo, el principal indicador de la Bolsa Mexicana de Valores había sufrido una pérdida acumulada del 8.1\%, (Fondo Monetario Internacional (FMI), 2007), mientras que el peso mexicano experimentó una depreciación respecto al dólar estadounidense del 1.3\% (Banxico, 1997). Aunque la volatilidad fue de corta duración, entre los inversionistas quedó la inquietud del curso que podrían seguir los acontecimientos ante una probable caída de la economía china.

A medida que se acrecienta el fenómeno de la globalización e integración de los mercados financieros, es importante continuar investigando sobre los elementos que puedan ayudar a evitar que el contagio se vuelva a repetir, incluso aunque éste sea cada vez menos frecuente.

Este artículo retoma el planteamiento de Didier et al. (2006) quienes afirman que la razón por la que el contagio financiero pareciera irse desvaneciendo se debe principalmente a la anticipación de las crisis financieras. De modo que una crisis como la de Argentina de 2001, que fue ampliamente anticipada por el constante y prolongado deterioro de las variables fundamentales, se espera haya tenido un efecto casi nulo sobre los mercados mundiales al tener los 
inversionistas tiempo de reducir su exposición al mercado argentino y por tanto, de rebalancear sus portafolios de manera paulatina.

En cambio, crisis no anticipadas deben ocasionar que el efecto contagio tenga un mayor impacto sobre el resto de las economías, debido a que los inversionistas tienen que liquidar de manera rápida sus activos financieros para rebalancear sus portafolios, lo que los lleva a realizar ventas en países ajenos a la crisis e incluso crear pánico entre los inversionistas menos informados. Tal fue el caso de la crisis mexicana de 1994 y de la asiática de 1997, cuyos efectos se dejaron sentir en otros mercados.

La crisis argentina de 2001 fue antecedida por acontecimientos que crearon en los inversionistas la expectativa de movimientos adversos en los mercados financieros de ese país, como fueron: una recesión prolongada, incremento de la deuda pública y privada, deterioro en la capacidad de pago de la deuda externa e interna, incapacidad en la obtención de créditos, falta de acción gubernamental y una advertencia del FMI en 1998 acerca de la posibilidad de que la economía sufriera un colapso similar al de los países del sudeste asiático (Bustelo, 2007; Daseking et al. 2005; Didier et al., 2006; Maletta, 2007).

Por el contrario, antes de que comenzaran las turbulencias financieras en el sudeste asiático en 1997 y durante el principio de las mismas, las condiciones y expectativas sobre el desempeño de los mercados financieros en Hong Kong eran opuestas a las que se tenían sobre Argentina. Las variables fundamentales de la economía de esa nación eran sostenibles y contaba con un sistema financiero y una política fiscal y monetaria eficiente que le permitía mantener la estabilidad del tipo de cambio (Kaminsky y Schmukler, 1999; Chen y Ng, 2000; Chul Park y Song, 1999; Yam, 1999).

El propósito de este artículo consiste en analizar el efecto contagio que una crisis anticipada (Argentina, 2001) y una no anticipada (Hong Kong, 1997) pudieron haber tenido sobre los mercados financieros de Brasil y México. Se escogen Brasil y México por ser dos de las economías más grandes y abiertas de la región de América Latina. Se considera la existencia de contagio cuando 
hay evidencia de un incremento significativo en la correlación de las variables financieras de un país que entra en crisis sobre las mismas variables de otro; de manera más específica, el contagio sobre los rendimientos accionarios y tasas de interés reales.

Se considera al canal financiero como el medio de transmisión de los movimientos en las variables financieras, ya que es por este canal donde la anticipación o no de las crisis puede causar movimientos grandes de capital, recomposición apresurada de carteras y efecto manada.

Esta investigación, ante el hecho de una falta de acuerdo sobre lo que implica el contagio financiero, retoma la definición propuesta por Edwards y Susmel (1999) que explica la existencia de contagio como un cambio inusual en la correlación de las variables entre dos o más países inducido por un choque externo, lo cual se estimará mediante un modelo similar al propuesto por Bekaert et al. (2005).

Los estudios que han utilizado este modelo han analizado la existencia de contagio de los índices accionarios entre bloques regionales, más que entre países individuales, para los períodos que abarcan la crisis mexicana de 1994 y la asiática de 1997. En este estudio los sujetos son diferentes a los encontrados en la revisión de la literatura. En un primer momento se considerará el efecto que la crisis de Hong Kong de 1997 pudo haber tenido sobre los mercados de Brasil y México, para posteriormente analizar el efecto que la crisis argentina de 2001 pudo tener sobre esos mismos mercados. Por otra parte, no sólo se considerará el rendimiento de los índices accionarios, sino también el comportamiento de las tasas de interés reales como variables de contagio.

La estructura de este estudio se compone de la siguiente manera: en la sección dos se hace una revisión de la literatura sobre el contagio financiero y un referente histórico acerca de los antecedentes que detonaron las crisis de Hong Kong en 1997 y de Argentina en 2001. La sección tres explica la hipótesis, el modelo, la metodología y los datos utilizados. La sección cuatro muestra la estadística descriptiva. En la sección cinco se presentan los resultados econométricos y en la sección seis se dan las conclusiones. 


\section{REVISIÓN DE LA LITERATURA}

\subsection{TEORÍAS SOBRE CONTAGIO}

Dentro de la literatura sobre el contagio financiero, no existe ningún consenso acerca de lo que éste representa y las características del mismo (Forbes y Rigobon, 2002; Boschi, 2005). En una aproximación básica algunos autores asumen que el contagio es aquel evento que ocurre cuando la extensión de la crisis en un país puede ser explicada por lo que ocurre en otra parte (Caramazza et al., 2004; Hernández y Valdez, 2001; Moser, 2003). No obstante, existe una discrepancia ideológica y metodológica para determinar si los eventos económicos y financieros de un país son efectivamente consecuencia de lo que ocurre en otro país.

Para estimar la existencia de contagio, algunos autores analizan la ocurrencia de incrementos significativos en la correlación de las variables de los mercados analizados, la cual tiene que darse después de que acontezca un evento económico o financiero negativo en un país o grupo de países (Eichengreen et al., 1996; Dornbusch et al., 2000; Forbes y Rigobon, 2002); o de manera más específica, este incremento en la correlación se tiene que dar durante un período considerado como de crisis (Calvo y Reinhart, 1996; Frankel y Schmukler, 1998; Baig y Goldfajn, 2000; Forbes y Rigobon, 2001). En un enfoque más restrictivo, Bekaert et al. (2005) proponen que para que el evento sea considerado como contagio no basta un incremento en la correlación, sino que debe haber evidencia de un exceso de correlación entre las variables analizadas.

Autores como Edwards y Susmel (1999) suponen que el contagio está relacionado con la volatilidad en los mercados e implica un cambio inusual y de corta duración en la misma; esta volatilidad es inducida por un choque externo y es explicada por los lazos regionales que a su vez originan relaciones de interdependencia entre los países. Sin embargo para Dungey et al. (2005), las etapas de contagio, además de verse relacionadas con un cambio en la volatilidad implican la aparición de correlaciones adicionales que no son explicadas por las simples relaciones de interdependencia entre las economías. 
Otro grupo de autores, independientemente de medir el incremento en la correlación y/o en la volatilidad de los mercados, suponen que el contagio también involucra el comportamiento de los inversionistas. De este modo, Bayoumi et al. (2003); Calvo (2005); Calvo y Mendoza (1999); Pritsker (2001); Kumar y Peraud (2002); Kodres y Pritsker (2002), definen contagio como aquel evento provocado por el comportamiento en manada, donde los inversionistas no informados seguirán las acciones de los inversionistas que supuestamente tienen mejor información. En una aproximación similar, Jaque (2004) explica que el contagio es un comportamiento negativo de una economía después de haberse dado exitosamente un ataque especulativo por parte de los inversionistas en otra economía, donde los países contagiados muestran generalmente variables fundamentales sólidas.

En cuanto a las variables más frecuentemente utilizadas para la estimación del contagio se encuentran: índices accionarios, tipos de cambio, tasas de interés, flujos de capital y/o diferenciales en deuda soberana. Estas variables se pueden utilizar de manera conjunta o individual (Kaminsky y Schmukler, 1999; Ganapolsky y Schmukler, 1998; Karolyi, 2003).

La literatura no únicamente encuentra discrepancias en la definición y el modo en que se estima la existencia de contagio, sino que también difiere sobre los canales mediante los cuales éste se puede transmitir. Los canales más frecuentemente utilizados para explicar el contagio son: las relaciones comerciales, devaluaciones competitivas y las relaciones financieras.

\subsection{RELACIONES COMERCIALES Y DEVALUACIONES COMPETITIVAS}

Dentro de este grupo de teorías se encuentra la propuesta de que el contagio es ocasionado por el grado de cercanía de los países a la región o país que experimenta la crisis original (Glick y Rose, 1999; Hernández y Valdez, 2001).

Las relaciones comerciales explican el contagio debido a la posibilidad de la ocurrencia de devaluaciones competitivas, las cuales ocasionan que las mercancías exportables se abaraten respecto a las de los competidores, aunque 
sea de manera temporal (Dornbusch et al., 2000; Hernández y Valdez, 2001; Forbes y Rigobon, 2001; Karoly, 2003). Esta situación presiona a otros países a ajustar el valor de sus monedas para evitar que las mercancías exportables sean desplazadas por los precios relativos más bajos en otros países (Gerlach y Smetts, 1995; Caramazza et al., 2004). Además, la distorsión en los precios que ocurre como resultado de una devaluación, crea la posibilidad de generar un ataque especulativo sobre el resto de las monedas de los principales socios comerciales, debido a que los inversionistas supondrán que éstos tendrán que ajustar el tipo de cambio para reestablecer el equilibrio comercial (Kumar y Peraud, 2002).

Por otra parte Forbes (2004) añade como causal de contagio el llamado efecto ingreso. Los ciudadanos de un país afectado por la devaluación ven reducida su capacidad de compra en términos de las monedas extranjeras, disminuyendo así la demanda por productos importados y ocasionando una disminución en las exportaciones de los países que no fueron afectados por la devaluación pero que mantienen relaciones comerciales fuertes con el país afectado. Esto da como resultado un deterioro de sus balanzas comerciales.

No obstante, Caramazza et al. (2004) encuentran que si bien el canal comercial es relevante al momento de explicar el contagio, han ocurrido episodios de este fenómeno donde el canal no es explicativo. Tal es el caso de la crisis rusa de 1998, que transmitió volatilidad financiera a otras regiones con las que no mantenía relaciones comerciales. Es así que otra corriente de la literatura considera a las relaciones financieras como un canal de mayor relevancia para la transmisión del contagio.

\subsection{RELACIONES FINANCIERAS}

Muchos analistas argumentan que los canales financieros han sido los principales propagadores de las crisis recientes, entre los que se encuentran Baig y Goldfajn (2000); Kaminsky et al. (2003); Van Rijckeghem y Weder (2001); Caramazza et al. (2000). Cuando estalla la crisis en un país, se originan repercusiones importantes en los mercados financieros locales (Forbes, 2004). Esto 
obliga a inversionistas nacionales y extranjeros a realizar movimientos (muchas veces fuertes) en otros mercados para recomponer sus inversiones.

Asimismo, cuando la integración financiera de un país en el mercado mundial se incrementa, la crisis se transmite de una manera más rápida debido a la facilidad con la que se mueven los capitales (Dornbusch et al., 2000).

Los cambios en los flujos de capital influyen de manera directa en la propensión de un país a caer en crisis. A continuación se analizan tres factores que pueden ocasionar que los inversionistas retiren sus fondos de mercados no localizados en el país que presenta inicialmente la crisis. Estos factores se agrupan dentro del apartado siguiente de anticipación de las crisis y expectativas de los inversionistas. Es importante señalar que los factores que se mencionan a continuación no son mutuamente excluyentes, ya que pueden ocurrir al mismo tiempo o bien uno puede ser generador de otro.

\subsubsection{ANTICIPACIÓN DE LAS CRISIS Y EXPECTATIVAS DE LOS INVERSIONISTAS}

Para Radelet y Sachs (1999), Feldstein (2002) y Stiglitz (1999), el cambio repentino en las expectativas es una fuente importante por la que se transmiten las crisis, ya que ocasiona variaciones en la confianza y comportamiento de los inversionistas. Esto afecta a los mercados e induce a la economía de los países vulnerables al contagio a moverse hacia un entorno adverso (Edwards y Susmel, 1999). La literatura acerca del contagio supone que los siguientes elementos son los causantes del cambio en el comportamiento de los agentes financieros:

“WAKE UP CALL”

Un cambio en los flujos de capital trae consigo presiones para que un país entre en crisis. Este cambio en los flujos se puede dar por el llamado wake up call, que ocurre cuando los inversionistas observan la debilidad en un país y se retiran de países con características similares (Goldstein, 1998; Forbes y Rigobon, 2001; Caramazza et al., 2004; Kaminsky et al., 2003; Forbes, 2004; Moser, 2003). 
Los parecidos macroeconómicos entre los países originan contagio si los inversionistas consideran que tienen una propensión similar a entrar en crisis. En estos casos los inversionistas realizarán movimientos similares a aquellos que hacen con el país en el que primero se detonó la crisis, creándoles problemas de liquidez (Valdez, 1997; Hernández y Valdez, 2001). Es así que los países con variables fundamentales débiles son vulnerables a sufrir un colapso de su moneda y de su sector financiero (Sachs et al., 1996; Edwards y Susmel, 1999).

Incluso aunque los países tengan variables fundamentales sólidas, la información que poseen los inversionistas suele ser imperfecta, lo que los lleva a una interpretación incorrecta de las variables fundamentales y, como consecuencia de ésta, a liquidar sus posiciones (Moser, 2003).

Bajo este contexto uno de los elementos principales para que se origine el contagio es la aparición sorpresiva de las crisis, particularmente luego de un período en el que las entradas de capital han sido considerables, ya que la interrupción o salida de capitales pueden crear problemas de liquidez en el corto plazo (Kaminsky et al., 2003).

\section{RECOMPOSICIÓN DE PORTAFOLIOS}

Existe interdependencia entre los mercados financieros, ya que se toman fondos de varios países para recomponer los portafolios a los niveles de riesgo y rendimiento deseados. Cuando hay una crisis financiera, los inversionistas tienden a cambiar su posesión de activos de alto riesgo por aquellos de bajo riesgo. Entre estos activos poco riesgosos están los papeles de gobierno de economías sólidas, lo que puede originar problemas de liquidez en aquellos países vulnerables al contagio (Moser, 2003). Es así que cuando aparecen problemas de liquidez en un mercado por una crisis, se extraen fondos de otros países, creando en éstos también problemas de liquidez (Hernández y Valdez, 2001). Incluso, los administradores de fondos pueden vender activos en varios mercados para financiar posibles retiros de los inversionistas luego de una crisis en un mercado en particular (Valdez, 1997; Calvo, 2005). 
Cuando una crisis no es anticipada, los agentes financieros se ven obligados a recomponer sus portafolios de manera apresurada (Valdés, 1997). La salida de capitales generalmente es detonada inicialmente por una cantidad pequeña de individuos, quienes eligen liquidar sus activos en aquellos mercados vulnerables a sufrir contagio (Dornbusch et al., 2000; Shleifer y Vishny, 1997). Una vez que se ha iniciado el evento de contagio, los mercados se encuentran con poca liquidez, lo cual puede obligar a los inversionistas (que tienen que enfrentar vencimientos anticipados) a liquidar sus activos a un precio muy bajo (Calvo, 2006).

Ante tal situación, Kodres y Pritsker (2002) afirman que incluso los inversionistas bien informados necesariamente tendrán que recomponer sus portafolios, vendiendo activos de otros mercados a precios bajos para disminuir su exposición al riesgo (o bien para obtener efectivo; Caramazza et al., 2004).

En estos casos, no solo los inversionistas liquidan activos de mercados inicialmente sanos; también los bancos de un deudor común inmerso en la crisis pueden sufrir un deterioro en la calidad de sus créditos. Con el fin de reducir el riesgo global, estos bancos tienden a disminuir su exposición a inversiones riesgosas colocadas en otros mercados (Dornbusch et al., 2000), así como sus líneas de crédito.

Por el contrario, una anticipación de las crisis otorga tiempo tanto a los inversionistas como a los bancos para recomponer sus portafolios de manera

paulatina y ordenada, evitando de este modo que sobrerreaccionen los precios de los activos ante movimientos fuertes de capital (Kaminsky et al., 2003).

\section{EFECTO MANADA}

Debido a que la información es costosa, algunos inversionistas siguen las acciones de otros agentes como política para la toma de decisiones. Esto se da siempre y cuando las ganancias marginales de generar información ellos mismos sean menores que las ganancias marginales de tomar la información generada por otros inversionistas (Calvo y Mendoza, 1999).

Lo anterior puede llevar al contagio de las crisis, mediante el efecto manada. Este supone que los agentes copian la información de otros inversionistas, 
siempre y cuando sea óptimo seguir el comportamiento de ellos (Bikhchandani, 1992; Kaminsky et al., 2003). Cuando la crisis financiera en un país origina venta de activos en otros países, puede llevar a los inversionistas a suponer que todos los mercados enfrentarán el mismo problema, y seguirán el comportamiento de la mayoría (Dornbusch et al., 2000). De ese modo el contagio se origina mediante el pánico financiero, ocasionando que la crisis en algún mercado sirva para coordinar las expectativas de los agentes hacia la liquidación de activos en otros mercados (Edwards y Susmel, 1999; Moser, 2003).

Bayoumi et al. (2003) analizan los períodos en que los inversionistas se mueven en manada, como una manera de explicar el mecanismo como se transmite el contagio a países con variables fundamentales sólidas. Este efecto contagio puede darse incluso sin lazos comerciales importantes o hasta nulos entre los mercados; Baig y Goldfjan (2000) suponen que este es el caso del contagio de Rusia hacia Brasil en 1998, el cual cruzó fronteras regionales y que se dio aún sin relaciones comerciales y financieras directas entre ambos países. Esto se conoce en la literatura como contagio irracional (Karoly, 2003); sin embargo, este comportamiento de los agentes no es necesariamente irracional, ya que el cambio en sus expectativas y acciones se da con la finalidad de disminuir el riesgo de sus portafolios.

\subsection{DETERMINACIÓN DE LOS CASOS}

La crisis de Hong Kong en 1997 fue inesperada, contrario a la ocurrida en Argentina en 2001. A continuación se analizan los elementos que permiten suponer que las expectativas de los inversionistas no incluían un deterioro significativo de la situación económica y financiera de Hong Kong en 1997, en contraste con las expectativas que se tenían acerca del comportamiento de la economía argentina antes de la crisis de octubre de 2001.

\subsubsection{EL CASO HONG KONG}

Chen y Ng (2000) argumentan que antes de la crisis del este asiático de 1997, las variables fundamentales de la economía de Hong Kong eran consideradas 
sólidas por parte de los inversionistas. Esta percepción se basaba principalmente en la apreciación general de que la economía del país contaba con un sistema financiero y una política fiscal y monetaria sustentable, que le permitía mantener niveles altos de crecimiento y estabilidad financiera $y$ macroeconómica.

Las instituciones bancarias de Hong Kong gozaban de prestigio en los mercados financieros mundiales, por mantener una política administrativa prudente y conservadora. Esto le daba solidez al sistema para resistir cambios fuertes en las posiciones financieras. Las autoridades monetarias mantenían un tipo de cambio fijo respecto al dólar de los Estados Unidos. Este era sustentado a través de un consejo monetario, considerado el más transparente de toda la región, lo que daba certidumbre a los inversionistas sobre el mantenimiento del tipo de cambio y la seguridad de sus inversiones (Yam, 1999).

El consejo monetario podía intervenir en el mercado cambiario al tener acceso a un gran volumen de reservas internacionales, cuyo monto era el tercero más grande de la región después de Japón y de China continental. Al comenzar los ataques especulativos estas reservas contabilizaban un total de 80 mil millones de dólares, aproximadamente cuatro veces el valor de M1 (FMI, 1999).

La literatura supone que la crisis asiática comenzó con la devaluación del baht de Tailandia el 2 de julio de 1997, trayendo consigo una serie de devaluaciones sobre las monedas de Filipinas, Malasia, Indonesia y Singapur. Esta serie de acontecimientos es denominada como la primera parte de la crisis asiática (Alba et al., 1998), donde mercados económicamente más sólidos de la región norte de la zona como Hong Kong, Japón, Taiwán, y Corea, no se vieron significativamente afectados por estos sucesos.

Sin embargo al agudizarse la situación financiera en los países del sudeste asiático, junto con desórdenes sociales y falta de acuerdos con el FMI, los mercados del norte comenzaron a sufrir ataques especulativos. La sorpresiva devaluación de la moneda de Taiwán, ocasionada por la decisión de las autoridades monetarias de no intervenir para mantener el tipo de cambio, creó dudas 
sobre el mantenimiento de la paridad de la moneda de Hong Kong (Kaminsky y Schmukler, 1999). Taiwán, aún con variables fundamentales sólidas, fue vulnerable ante la crisis del sudeste asiático; por ende, los inversionistas concluyeron que ni Hong Kong ni Corea serían inmunes a los choques adversos (Chul Park y Song, 1999).

La crisis financiera en Hong Kong comenzó el 17 de octubre de 1997, con un primer ataque especulativo masivo sobre su moneda y el desplome de la Bolsa de Valores de dicha región. Forbes y Rigobon (2002) señalan que éste es el detonante de la segunda parte de la crisis del este asiático, al cual le siguieron co-movimientos a nivel regional y mundial, así como una gran cantidad de publicidad negativa que proponía un evento mayor de contagio internacional.

Ante el ataque especulativo, las autoridades monetarias respondieron inyectando a la economía cinco mil millones de dólares de las reservas, incrementando las tasas de interés y comprando activos en la Bolsa de Valores de Hong Kong (BVHK) con valor de mil millones de dólares (Reyes, 1999). Si bien la intervención de las autoridades monetarias contuvo el ataque especulativo y se mantuvo la paridad de la moneda, las tasas de interés permanecieron volátiles durante muchos meses, la BVHK comenzó un importante retroceso y al final la economía entró en recesión.

Chul Park y Song (1999) sugieren que los acontecimientos en Hong Kong ocurrieron por un comportamiento en manada de los inversionistas ante la presencia de información asimétrica. Por otro lado, Radelet y Sachs (1999); Feldstein (2002); Stiglitz (1999) argumentan que la fuente primaria de la crisis fue el cambio repentino en las expectativas del mercado y en la confianza de los inversionistas. Corsetti et al. (1999) añade que el efecto pánico también pudo haber generado fuerzas para detonar la crisis.

En síntesis, las fugas masivas de capitales y el comportamiento de los inversionistas indican que la mayoría de éstos no anticiparon movimientos adversos fuertes en los mercados financieros de Hong Kong. Por lo tanto, sus expectativas se ajustaron de manera muy rápida y abrupta, con poca información disponible. 
EFECTOS DE LAS CRISIS ANTICIPADAS Y NO ANTICIPADAS SOBRE EL CONTAGIO FINANCIERO INTERNACIONAL

\subsubsection{ELCASO ARGENTINA}

Mientras que la crisis de Hong Kong (1997) posiblemente trajo un efecto contagio significativo, la que experimentó Argentina en 2001 parece haber quedado contenida dentro de las fronteras nacionales (con excepción de Uruguay), debido a que los mercados ya habían asignado con mucho tiempo de anticipación una alta probabilidad de que Argentina sufriera un colapso económico y financiero (Bustelo, 2007).

Argentina venía atravesando un período de recesión y deflación, el cual algunos autores denominan el "build up" de la crisis (ver Feldstein, 2002; Perry y Serven, 2002; Geithner, 2003). Al estallar la crisis financiera (cuyo mayor efecto se da en diciembre de 2001), las variables financieras y económicas sufrieron cambios muy fuertes respecto al período anterior. El pánico bancario ocasionó la adopción del llamado "corralito", el cual dejó sin liquidez a la economía. El fin del plan de convertibilidad trajo consigo una devaluación de facto del 40\%, que llegó a ser del 390\% en junio de 2002, al adoptarse el tipo de cambio libre. Ante estos movimientos, la inflación mensual alcanzó niveles del $10 \%$ en abril de 2002, presionando a las tasas de interés a niveles del 90\%; el Producto Interno Bruto experimentó un retroceso del 11\% y el gobierno dejó de pagar 90 millones de dólares por concepto del servicio de la deuda externa.

La caída continua y de largo plazo que presentó la Bolsa de Valores de Buenos Aires puede interpretarse como una anticipación por parte de los agentes de la posibilidad de movimientos adversos. De hecho, los fondos mutuos comenzaron a disminuir su exposición en el mercado argentino mucho antes de que estallara la crisis (situación que no se observó en otros episodios de crisis financieras de los años noventa), dando a los inversionistas tiempo de recomponer sus portafolios (Didier et al., 2006).

A partir de 1998 la expectativa de crecimiento de la economía argentina fue disminuyendo. Al mismo tiempo, los mercados financieros incrementaron la prima por riesgo y la tasa de interés líder que pasó de 6,5\% a 8,5\% (Banco Central de la República Argentina: BCA, 2007). Sin embargo, las entradas de 
capitales no mostraron incrementos significativos, lo que llevó a una menor inversión interna y a una disminución en la capacidad de los prestamistas para otorgar créditos (Moreno, 2002).

Entre enero y noviembre de 2001 el rendimiento de los bonos argentinos se incrementó en más de 20 puntos, colocándose en el 35\%, lo que muestra la adversidad de los inversionistas por este tipo de bonos (FRBSF, 2002). Como consecuencia hubo una mayor disminución en la inversión privada, mayores restricciones al crédito y una caída adicional en la expectativa de crecimiento (Daseking et al. 2005).

El tipo de cambio fijo, junto con la falta de incremento en la productividad, encarecía el precio relativo de los productos argentinos, deteriorando la balanza comercial y limitando aún más la entrada de divisas (Daseking et al. 2005). De la Torre et al. (2002) argumentan que el programa de convertibilidad argentino fue dejando sin liquidez a la economía ante la falta de divisas, lo cual derivó en deflación y desempleo, y erosionó la capacidad de repago de los agentes deudores, principalmente el gobierno.

La crisis financiera no fue ni sorpresiva ni causada por falta de información, sino por la incapacidad de implementar políticas que revirtieran el proceso de deterioro de las variables fundamentales. El déficit fiscal seguía una tendencia a la alza desde 1997 y aumentaba la incapacidad de liquidar la deuda interna; la razón de deuda pública a producto interno bruto (PIB) pasó del 35\% en 1995 al $65 \%$ en el 2001, y se esperaba que siguiera creciendo debido a la incapacidad del sector público de incrementar la recaudación de impuestos, como consecuencia de la recesión prolongada.

El hecho de que la deuda se encontraba denominada en moneda extranjera, añadía al país un riesgo adicional ante un cambio en las tasas de interés externas o una depreciación de la moneda local. La razón de deuda externa a PIB pasó del $44 \%$ en 1990 a $51 \%$ en 2001, mientras que la razón deuda externa a exportaciones se encontraba en niveles del $470 \%$ en el año 2000 , con tendencia al alza debido a la continua disminución de las exportaciones (FRBSF, 2002). 
EFECTOS DE LAS CRISIS ANTICIPADAS Y NO ANTICIPADAS SOBRE EL CONTAGIO FINANCIERO INTERNACIONAL

Muchos analistas argumentaban que se estaba gestando una crisis financiera (Maletta, 2007). Si bien no se sabía con certidumbre la fecha en que Argentina tendría que ajustar sus políticas, los inversionistas ya habían comenzado desde tiempo atrás a adaptar sus expectativas. Cuando estalla la crisis, éstos no son tomados por sorpresa; dada la información previa, habían disminuido su exposición en el mercado argentino, lo cual no los obligó a realizar transacciones de gran volumen, como sucedió en el caso de Hong Kong.

\section{METODOLOGÍA}

Este estudio define al contagio como un aumento significativo en la correlación (que puede ser positiva o negativa) entre las variables económicas y/o financieras de dos o más países, inducido por un choque externo (Edwards y Susmel, 1999). Los sujetos de estudio son: Argentina, Brasil, México y Hong Kong, y las variables a utilizar son los rendimientos de los índices accionarios y las tasas de interés reales.

El modelo econométrico que se propone, tiene la finalidad de analizar el modo en que la crisis argentina de 2001 (considerada como anticipada) afectó a Brasil y México, para posteriormente comparar sus efectos con los de la crisis de Hong Kong de 1997 (sobre Brasil y México), la cual no era prevista por la mayoría de los agentes financieros.

\subsection{ESPECIFICACIÓN DEL MODELO}

Para verificar la existencia de contagio primero se analizará el exceso de correlación de los rendimientos accionarios, con una versión modificada del modelo de Bekaert et al. (2005). En éste, el exceso en la correlación se observa al comparar períodos estables, con períodos en los que los países que pudieran originar el contagio se encuentran en crisis. Posteriormente, el mismo método se utilizará para analizar el comportamiento de las tasas de interés reales de los países sujetos de estudio. 
El modelo inicial es el siguiente (para rendimientos accionarios, pero es análogo para tasas de interés reales):

$$
\hat{\mathrm{e}}_{\mathrm{b}, \mathrm{t}}=\mathrm{a}_{\mathrm{b}}+\mathrm{b}_{\mathrm{b}, \mathrm{t}} \hat{\mathrm{e}}_{\mathrm{a}, \mathrm{t}}+\mu_{\mathrm{b}, \mathrm{t}}
$$

con:

$$
b_{b, t}=b_{0}+b_{1} D_{1}
$$

Donde:

$\hat{e}_{b, t}$ Es el rendimiento del índice accionario del país que es sujeto a contagio expresado como $\ln \mathrm{p}_{\mathrm{t}+1}-\ln \mathrm{p}_{\mathrm{t}}$, donde $\mathrm{p}_{\mathrm{t}}$ es el valor de cierre (en el tiempo $\mathrm{t}$ ) del índice accionario representativo de la bolsa de valores de este país.

$\mathrm{a}_{\mathrm{b}} \quad$ Es el término constante, que es independiente del comportamiento del mercado accionario del país que pudiera originar el contagio. Este término constante puede reflejar choques externos comunes que afectarían el comportamiento accionario de todos los mercados, así como elementos de la economía local.

$\hat{e}_{a, t}$ Es el rendimiento del índice accionario del país que origina el contagio, expresado también como $\ln \mathrm{p}_{\mathrm{t}+1}-\ln \mathrm{p}_{\mathrm{t}}$

$\mu_{\mathrm{b}, \mathrm{t}}$ Es el término de error aleatorio.

$b_{b, t}$ Es el coeficiente que indica el modo cómo se relaciona el rendimiento accionario del país que origina el contagio con el rendimiento accionario del país sujeto a contagio.

Como se aprecia en la ecuación (2), el coeficiente $b_{b, t}$ se puede descomponer en dos partes: $b_{0}$ se utiliza para estimar la relación (en períodos tranquilos) de los rendimientos accionarios de los dos países, mientras que $\mathrm{b}_{1} \mathrm{D}_{1}$ indica esta relación en los períodos en que el país que origina el contagio se encuentra en crisis.

$\mathrm{D}_{1}$ es una variable dummy, que toma el valor de 1 cuando el país que origina el contagio está en crisis (cero en los demás casos). 
Es probable que el contagio sobre el rendimiento accionario y/o las tasas de interés reales de México y/o Brasil no se presente en el mismo momento en que se da la crisis financiera en Argentina y/o Hong Kong, sino a posteriori. Para capturar esta posibilidad, la ecuación (1) se amplía de la siguiente manera:

$$
\hat{\mathrm{e}}_{\mathrm{b}, \mathrm{t}+\mathrm{i}}=\mathrm{a}_{\mathrm{b}}+\mathrm{b}_{\mathrm{b}, \mathrm{t}} \hat{\mathrm{e}}_{\mathrm{a}, \mathrm{t}}+\mu_{\mathrm{b}, \mathrm{t}}, \text { con } \mathrm{i}=0,1 \ldots
$$

En la ecuación (3), i se refiere al número de períodos de adelanto para el rendimiento del índice accionario (o en su caso, para la tasa de interés real) del país que es sujeto a contagio.

Es importante señalar que este estudio no intenta pronosticar el valor de los rendimientos accionarios (o de las tasas de interés reales) de México y Brasil, sino la relación que existe entre estos y los de Argentina y Hong Kong, durante períodos tranquilos y de crisis. Por tanto, el análisis de contagio se enfoca en el valor y significancia de los coeficientes $b_{b, t}, b_{0} y_{1}$. No obstante, dado que existen variables que pudieran estar afectando esta relación, como análisis de sensibilidad se reconoce la necesidad de estimar un modelo estructural.

De acuerdo con el teorema de Gauss-Markov, para un modelo lineal cuyos errores esperados son iguales a cero, no están correlacionados y además tienen la misma varianza; el mejor estimador lineal no sesgado de los coeficientes es aquel estimado mediante mínimos cuadrados ordinarios. Sin embargo, las series de tiempo de variables financieras que presentan períodos de volatilidad generalmente son heteroscedásticas; es decir, la varianza de los errores no es constante. Además, la media de los errores no es igual a cero, por lo que no se cumple con los supuestos básicos de la estimación eficiente de parámetros mediante mínimos cuadrados ordinarios.

Por ende, para tener un mejor estimador del modelo se utilizará el método Newey-West, el cual es un procedimiento que genera parámetros que son consistentes en presencia de autocorrelación y de heteroscedasticidad (Greene, 1997).

Para probar la hipótesis de contagio ( $\left|b_{1}\right|>\left|b_{0}\right| ;\left|b_{1}\right| \neq 0$ ) se utiliza la prueba Wald, la cual permite analizar qué tan bien los coeficientes estimados 
en una regresión sin restricciones satisfacen las restricciones de la hipótesis planteada. Se toma la decisión de rechazar la hipótesis con un intervalo de confianza del $10 \%$.

\subsection{HIPÓTESIS}

Cuando una crisis es anticipada, los agentes financieros han tenido tiempo de recomponer sus carteras ante la eventual ocurrencia del evento. De llegar a estallar la crisis, los portafolios ya tienen incorporado el nivel de riesgo deseado por los inversionistas. Incluso, éstos han tomado las medidas preventivas necesarias para aminorar el efecto de las crisis sobre los mismos. Por ende, los efectos contagio de una crisis financiera anticipada versus una no anticipada deben ser menores.

Se parte de que a lo largo del tiempo existe una relación entre los rendimientos de los índices accionarios y tasas de interés reales del país que presentó crisis y aquel sujeto al contagio: $\mathrm{H}_{01}: \mathrm{b}_{\mathrm{b}, \mathrm{t}} \neq 0$.

Se procede a determinar si la relación entre los rendimientos de los índices accionarios y tasas de interés reales es distinta durante períodos tranquilos y de crisis; se espera que la correlación en períodos de crisis aumente, lo cual indica contagio, es decir $\mathrm{H}_{02}:\left|\mathrm{b}_{1}\right|>\left|\mathrm{b}_{0}\right| ;\left|\mathrm{b}_{1}\right| \neq 0$.

El signo del coeficiente es importante para determinar el tipo de contagio. Un coeficiente $b_{1}$ negativo indica una relación inversa entre los rendimientos accionarios (o tasas de interés reales), lo cual implica un contagio positivo; es decir, el país sujeto a contagio experimenta un efecto positivo debido a la crisis en el país que origina el contagio.

$\mathrm{Si}_{1}$ es positivo y significativo, entonces hay correlación directa entre los mercados, lo que muestra contagio negativo; es decir, el país sujeto a contagio se ve afectado de manera adversa por el país que origina la crisis.

Para probar que la anticipación de las crisis disminuye el efecto contagio, debe darse que el coeficiente $b_{1}$ sea menos significativo en el caso de la crisis de Argentina que en la crisis de Hong Kong. 
EFECTOS DE LAS CRISIS ANTICIPADAS Y NO ANTICIPADAS SOBRE EL CONTAGIO FINANCIERO INTERNACIONAL

\subsection{DEFINICIÓN DE PERÍODOS DE CRISIS Y PERÍODOS NORMALES}

Baele e Inghelbrecht (2006) realizan una recopilación de los distintos períodos de crisis que han utilizado varios autores en artículos relacionados con el tema de contagio. Retomando dicha información para el caso de Hong Kong, el período de crisis transcurre del 17 de octubre de 1997 (fecha en que inician los ataques especulativos contra la moneda de esa nación) al 28 de enero de 1998, cuando los mercados financieros comienzan a recuperarse tras formalizar negociaciones claves con el FMI.

Con el fin de evitar que la información se contamine por el contagio ocasionado por otras crisis, el primer período tranquilo transcurre del 16 de octubre de 1996 (donde las variables macroeconómicas mexicanas están estables después de la crisis de 1995) al 16 de octubre de 1997. El segundo período tranquilo es de menor duración: del 29 de enero de 1998 al 23 de marzo de 1998, fecha en que el presidente Boris Yeltsin de Rusia despide a todo su gabinete económico y empieza la especulación contra la moneda rusa, lo que ocasiona movimientos adversos en los mercados mundiales.

Para el caso argentino, el período de crisis inicia el 29 de octubre de 2001 (aunque es en diciembre cuando se ve el mayor efecto), fecha en que comienza la salida masiva de fondos de las instituciones bancarias de esa nación. Este termina el 31 de agosto de 2002, cuando los mercados dan señales de estabilización (Geithner, 2003).

En contraste con el caso de Hong Kong, donde la crisis mexicana y la crisis rusa ocasionaron ruido y acortaron los períodos tranquilos, para Argentina es posible capturar una mayor cantidad de información (dos años antes y después de la crisis). En este lapso de tiempo no existe evidencia de ningún evento que haya ocasionado contagio; por lo tanto, el primer período tranquilo va del 28 de octubre de 1999 al 28 de octubre de 2001 y el segundo período tranquilo parte del 1 de septiembre de 2002 al 1 de septiembre de 2004. 


\subsection{FUENTE DE DATOS}

La fuente primaria de los datos diarios de cierre de los índices accionarios proviene de las Bolsas de Valores de Argentina (Índice Merval), Brasil (Índice Bovespa), Hong Kong (Índice Hang Seng) y México (Índice de Precios y Cotizaciones). La información sobre las tasas de interés reales emana de los Bancos Centrales de Argentina (Tasa de Referencia a 30 días), Brasil (Central Bank Prime Rate a 30 días), Hong Kong (Treasury Bonds a 30 días) y México (Certificados de la Tesorería a 28 días).

Para homogeneizar la información utilizada en este estudio, el valor diario de cierre de los índices bursátiles de las Bolsas de Valores de Argentina, Brasil, Hong Kong y México se recopiló de las estadísticas financieras del FMI. El valor promedio mensual de las tasas de interés reales líder para cada uno de los cuatro países mencionados anteriormente se obtuvo también de la misma fuente.

El FMI provee información a través del Boletín de Diseminación Estándar (SDDS), el cual sigue una serie de códigos y lineamientos para que los datos sean presentados de manera estandarizada y por tanto sean comparables.

\section{ESTADÍSTICA DESCRIPTIVA}

\subsection{COMPORTAMIENTO DE LOS RENDIMIENTOS ACCIONARIOS DE BRASIL, HONG KONG Y MÉXICO}

La tabla 1 muestra el valor de la media, el mínimo, el máximo y la desviación estándar de los rendimientos accionarios de los índices de las bolsas de valores de Brasil, Hong Kong y México, para los períodos antes, durante y después de la crisis de Hong Kong de 1997. Se aprecia que los valores durante la crisis muestran un comportamiento distinto en comparación con los observados en otros períodos. 
EFECTOS DE LAS CRISIS ANTICIPADAS Y NO ANTICIPADAS SOBRE EL CONTAGIO FINANCIERO INTERNACIONAL

TABLA 1

Comportamiento de los rendimientos accionarios diarios de Brasil, Hong Kong y México en los períodos de pre crisis, crisis y post crisis en Hong Kong (\%)

\begin{tabular}{|l|l|c|c|c|}
\hline & & $\begin{array}{c}\text { Pre crisis } \\
10 / 16 / 96- \\
10 / 16 / 97\end{array}$ & $\begin{array}{c}\text { Crisis } \\
10 / 17 / 97- \\
01 / 28 / 98\end{array}$ & $\begin{array}{c}\text { Post crisis } \\
01 / 29 / 98- \\
03 / 23 / 98\end{array}$ \\
\hline \multirow{4}{*}{ Media } & Brasil & 0.37 & -0.13 & 0.56 \\
\cline { 2 - 5 } & Hong Kong & 0.14 & -0.51 & 0.63 \\
\cline { 2 - 5 } & México & 0.17 & -0.10 & 0.28 \\
\hline \multirow{4}{*}{ Mánimo } & Brasil & -3.50 & -16.22 & -1.53 \\
\cline { 2 - 5 } & Hong Kong & -3.74 & -14.73 & -4.94 \\
\cline { 2 - 5 } & México & -2.80 & -14.31 & -2.14 \\
\hline \multirow{3}{*}{ D. estándar } & Brasil & 6.78 & 9.26 & 2.57 \\
\cline { 2 - 5 } & Hong Kong & 4.37 & 17.25 & 13.39 \\
\cline { 2 - 5 } & México & 3.62 & 11.06 & 3.02 \\
\cline { 2 - 5 } & Brasil & 1.36 & 3.72 & 1.08 \\
\cline { 2 - 5 } & Hong Kong & 1.11 & 3.96 & 2.89 \\
\cline { 2 - 5 } & México & 1.03 & 2.45 & 1.30 \\
\hline
\end{tabular}

El rendimiento de cada índice se estimó como $1 n \mathrm{p}_{\mathrm{t}+1}-\ln \mathrm{p}_{\mathrm{t}}$, siendo $\mathrm{p}_{\mathrm{t}}$ el valor de cierre (en el tiempo t) del índice accionario representativo de cada bolsa de valores.

Fuente: Elaboración propia con base en información de las bolsas de valores de los países en cuestión.

Durante el período de crisis los tres países presentan rendimientos medios accionarios negativos, siendo Hong Kong el que experimenta la caída más fuerte. Ya que en el período pre crisis los tres mercados presentan rendimientos accionarios positivos, se concluye que la crisis de Hong Kong afectó de manera negativa a todos los mercados analizados. En el período post crisis se recuperan los 3 mercados, en especial el de Hong Kong, con rendimientos accionarios promedio muy superiores al período pre-crisis. En Hong Kong, esto puede ser consecuencia de una subvaluación previa del precio de los activos financieros, aunado con la disminución en las tasas de interés posterior a la crisis, y el ligero optimismo por parte de los inversionistas ante los acuerdos 
alcanzados con los organismos financieros internacionales; sin embargo, es difícil hacer inferencias debido al corto período post crisis considerado.

Los valores máximos, mínimos y la desviación estándar del rendimiento accionario de todos los mercados se ven incrementados durante el período de crisis de Hong Kong. Esto muestra que hay una mayor fluctuación en estos mercados durante esta crisis, al compararla con los otros períodos estudiados. Como es de esperar, la volatilidad es mayor para Hong Kong que para los demás.

En el período posterior a la crisis, la fluctuación de los rendimientos (medida en términos de la desviación estándar) presenta una disminución en los tres mercados, siendo la caída en Brasil y México en términos porcentuales mayor a la de Hong Kong. Esto podría implicar que los factores que ocasionaron la crisis en Hong Kong permanecían afectando la volatilidad de este mercado, pero ya no originaban volatilidad sobre los mercados brasileño y mexicano.

La tabla 2 muestra el coeficiente de correlación de los rendimientos accionarios para cada par de países en los 3 períodos de estudio. Se puede observar que durante el período de crisis de Hong Kong, hay un incremento en la correlación de todos los mercados. Este aumento es particularmente significativo para Brasil y México, lo que sugiere que los inversionistas no discriminaron entre los países de la región latinoamericana y retiraron fondos de ambas naciones ante los eventos en Hong Kong.

No obstante, durante el período de crisis se puede observar que la correlación del mercado brasileño con el de Hong Kong presenta un incremento mayor (475\%), en comparación con el aumento en la correlación del mercado de Hong Kong con el mexicano (20\%). Esto sugiere que el mercado brasileño posiblemente experimentó un episodio más drástico de contagio que el mexicano.

Posterior a la crisis, se vuelve a incrementar la correlación del mercado de Hong Kong con los de México y Brasil. Es posible que el deterioro en la confianza de los inversionistas en el mercado de Hong Kong, pusiera a un nivel similar a este mercado con sus contrapartes en América Latina. Por otro lado, la correlación del mercado brasileño y mexicano disminuye, lo que sugiere que una vez reacomodadas las carteras, los inversionistas vuelven a diferenciar entre ambos mercados. 


\section{TABLA 2}

Comportamiento de los coeficientes de correlación de los rendimientos accionarios de Brasil, Hong Kong y México en los períodos de pre crisis, crisis y post crisis en Hong-Kong

\begin{tabular}{|l|c|c|c|}
\hline & $\begin{array}{c}\text { Pre crisis } \\
10 / 16 / 96- \\
10 / 16 / 97\end{array}$ & $\begin{array}{c}\text { Crisis } \\
10 / 17 / 97- \\
01 / 28 / 98\end{array}$ & $\begin{array}{c}\text { Post crisis } \\
01 / 29 / 98- \\
03 / 23 / 98\end{array}$ \\
\hline Hong Kong - Brasil & 0.04 & 0.23 & 0.31 \\
\hline Hong Kong - México & 0.15 & 0.18 & 0.33 \\
\hline Brasil - México & 0.03 & 0.67 & 0.34 \\
\hline
\end{tabular}

El coeficiente de correlación se estimó utilizando el procedimiento producto-momento de Pearson. Fuente: Elaboración propia con base en información de las bolsas de valores de los países en cuestión.

\subsection{COMPORTAMIENTO DE LOS RENDIMIENTOS ACCIONARIOS DE ARGENTINA, BRASIL Y MÉXICO}

La tabla 3 muestra el valor de la media, el mínimo, el máximo y la desviación estándar de los rendimientos accionarios de los índices de las bolsas de valores de Argentina, Brasil y México, para los períodos antes, durante y después de la crisis Argentina de 2001. Para todos los períodos, los estadísticos de Brasil y México muestran un patrón diferente respecto de los observados en Argentina.

Se nota un comportamiento atípico en el rendimiento promedio positivo de la bolsa de Argentina durante la crisis. Esto puede ser consecuencia de las restricciones impuestas por las autoridades monetarias argentinas, que desincentivaban en este período la inversión de fondos en la banca y dejaban a la bolsa como la única opción viable de inversión que pudiese volverse líquida (De la Torre et al., 2002). Sin embargo, no se puede descartar que los inversionistas hayan colocado fondos en la bolsa al anticipar o filtrarse información sobre las medidas en materia monetaria que podía tomar el gobierno argentino. 
TABla 3

Comportamiento de los rendimientos accionarios diarios de Argentina, Brasil y México

en los períodos de pre crisis, crisis y post crisis en Argentina (\%)

\begin{tabular}{|c|c|c|c|c|}
\hline & & $\begin{array}{l}\text { Pre crisis } \\
10 / 28 / 99- \\
10 / 28 / 01\end{array}$ & $\begin{array}{c}\text { Crisis } \\
10 / 29 / 01- \\
08 / 31 / 02\end{array}$ & $\begin{array}{l}\text { Post crisis } \\
09 / 01 / 02- \\
09 / 01 / 04\end{array}$ \\
\hline \multirow{3}{*}{ Media } & Argentina & -0.18 & 0.27 & 0.20 \\
\hline & Brasil & -0.02 & -0.04 & 0.17 \\
\hline & México & 0.00 & 0.06 & 0.10 \\
\hline \multirow{3}{*}{ Mínimo } & Argentina & -9.07 & -11.29 & -9.02 \\
\hline & Brasil & -7.54 & -6.75 & -6.34 \\
\hline & México & -8.27 & -4.51 & -5.44 \\
\hline \multirow{3}{*}{ Máximo } & Argentina & 7.84 & 16.12 & 6.49 \\
\hline & Brasil & 7.33 & 6.60 & 6.15 \\
\hline & México & 6.14 & 3.77 & 3.78 \\
\hline \multirow{3}{*}{ D. estándar } & Argentina & 2.23 & 3.96 & 2.04 \\
\hline & Brasil & 2.03 & 2.10 & 1.84 \\
\hline & México & 1.93 & 1.35 & 1.05 \\
\hline
\end{tabular}

El rendimiento de cada índice se estimó como $\ln \mathrm{p}_{\mathrm{t}+1}-\ln \mathrm{p}_{\mathrm{t}}$, siendo $\mathrm{p}_{\mathrm{t}}$ el valor de cierre (en el tiempo t) del índice accionario representativo de cada bolsa de valores.

Fuente: Elaboración propia con base en información de las bolsas de valores de los países en cuestión.

En el período anterior a la crisis de Argentina y durante la misma, Brasil y México siguen patrones diferentes en cuanto a la tendencia de los rendimientos accionarios medios, siendo en Brasil negativos y en México positivos. Sin embargo, el cambio absoluto en los rendimientos accionarios durante la crisis es tan pequeño que en una primera aproximación pareciera que la crisis argentina no alteró el comportamiento que venían siguiendo estos mercados (pudiéndose descartar la existencia de contagio).

En el período posterior a la crisis, el rendimiento medio de las acciones de la bolsa de Argentina experimenta una ligera disminución respecto al rendimiento observado durante el período de crisis. Esto podría ser consecuencia del levantamiento de las restricciones a la disponibilidad de efectivo impuestas 
durante el período de crisis, así como del incremento en las tasas de interés nominales (las cuales pasaron del 30\% en el mes anterior a la crisis a niveles del 100\% en agosto de 2002), lo que incentivó a un cambio en el destino de las inversiones.

Contrario a lo observado en Argentina, durante la etapa posterior al período de crisis, el rendimiento promedio de las bolsas de Brasil y México muestra un crecimiento. Esto, más que una respuesta a los cambios en la situación económica argentina, puede ser interpretado como un choque común en el que las bolsas mundiales iniciaron un comportamiento alcista una vez terminada la recesión en los Estados Unidos.

El mercado más volátil en todos los períodos, y en especial durante la crisis, es Argentina. Por el contrario, el mercado accionario en México presenta una tendencia a la baja en cuanto a su volatilidad; esto sugiere un comportamiento independiente a los acontecimientos en Argentina. Por su parte, las fluctuaciones en la bolsa de Brasil son pequeñas durante los tres períodos, lo cual indica también un comportamiento independiente a lo que ocurría en Argentina.

La tabla 4 muestra el coeficiente de correlación de los rendimientos accionarios para cada par de países, en los 3 períodos de estudio. Se puede

\section{TABLA 4}

Comportamiento de los coeficientes de correlación de los rendimientos accionarios de Argentina, Brasil y México en los períodos de pre crisis, crisis y post crisis en Argentina

\begin{tabular}{|l|c|c|c|}
\hline & $\begin{array}{c}\text { Pre crisis } \\
10 / 28 / 99- \\
10 / 28 / 01\end{array}$ & $\begin{array}{c}\text { Crisis } \\
10 / 29 / 01- \\
08 / 31 / 02\end{array}$ & $\begin{array}{c}\text { Post crisis } \\
09 / 01 / 02- \\
09 / 01 / 04\end{array}$ \\
\hline Argentina - Brasil & 0.56 & 0.09 & 0.29 \\
\hline Argentina - México & 0.43 & 0.11 & 0.24 \\
\hline Brasil - México & 0.01 & 0.04 & 0.27 \\
\hline
\end{tabular}

El coeficiente de correlación se estimó utilizando el procedimiento producto-momento de Pearson. Fuente: Elaboración propia con base en información de las bolsas de valores de los países en cuestión. 
apreciar la fuerte disminución de la correlación de los mercados brasileño y mexicano con el argentino durante el período de crisis de esta última nación. Esto da indicación de la falta de contagio internacional de la crisis en Argentina. En el período posterior a la crisis, la correlación entre las bolsas de los tres países es muy similar, lo que refleja el reestablecimiento del componente regional en las expectativas de los inversionistas. No obstante, empieza a verse una pequeña diferenciación relativa entre el mercado argentino y el mexicano.

\subsection{COMPORTAMIENTO DE LAS TASAS DE INTERÉS REALES DE BRASIL, HONG KONG Y MÉXICO}

La tabla 5 muestra el valor de la media, el mínimo, el máximo y la desviación estándar de las tasas de interés reales de Brasil, Hong Kong y México, para los períodos antes, durante y después de la crisis de Hong Kong de 1997. Se observa que durante el período de crisis estos valores aumentan en comparación con el período pre crisis.

Lo anterior sugiere, al igual que al tomar en cuenta el comportamiento de los índices accionarios, una situación de contagio financiero. Al incrementarse las tasas de interés reales en Hong Kong, los otros países respondieron con movimientos similares en sus propias tasas. Esto pudiera ser consecuencia de competencia en la captación de fondos y/o para evitar la salida de los mismos.

El país que presentó un aumento mayor en su tasa de interés real promedio fue Hong Kong (como es esperable). A este le siguió Brasil (138\%) y luego México (con un aumento del 72\%). Esto es consistente con el análisis anterior, donde se concluye que el mercado que posiblemente experimentó un episodio más drástico de contagio fue el brasileño.

En el período post crisis se aprecia una reducción en las tasas de interés reales promedio de los tres países. Esta señal de estabilización, junto a la recuperación que presentan los rendimientos de los índices accionarios, indica que el efecto contagio tiende a desaparecer. No obstante, debido a la poca información disponible en el período post crisis, es recomendable tomar con cautela esta conclusión. 
EFECTOS DE LAS CRISIS ANTICIPADAS Y NO ANTICIPADAS SOBRE EL CONTAGIO FINANCIERO INTERNACIONAL

\section{TABLA 5}

Comportamiento de las tasas de interés reales de Brasil, Hong Kong y México en los períodos de pre crisis, crisis y post crisis en Hong Kong (\%)

\begin{tabular}{|l|l|c|c|c|}
\hline & & $\begin{array}{c}\text { Pre crisis } \\
10 / 96-09 / 97\end{array}$ & $\begin{array}{c}\text { Crisis } \\
10 / 97-01 / 98\end{array}$ & $\begin{array}{c}\text { Post crisis } \\
02 / 98-03 / 98\end{array}$ \\
\hline \multirow{3}{*}{ Media } & Brasil & 11.77 & 28.07 & 24.09 \\
\cline { 2 - 5 } & Hong Kong & -0.61 & 3.07 & 1.13 \\
\cline { 2 - 5 } & México & 1.83 & 3.15 & 2.94 \\
\hline \multirow{3}{*}{ Mánimo } & Brasil & 7.78 & 15.22 & 18.56 \\
\cline { 2 - 5 } & Hong Kong & -2.11 & 2.19 & 1.09 \\
\cline { 2 - 5 } & México & 0.14 & 1.50 & 2.47 \\
\hline \multirow{3}{*}{ D. estándar } & Brasil & 14.58 & 35.19 & 29.47 \\
\cline { 2 - 5 } & Hong Kong & 0.69 & 4.16 & 1.19 \\
\cline { 2 - 5 } & México & 4.69 & 6.12 & 3.41 \\
\cline { 2 - 5 } & Brasil & 2.04 & 8.80 & 5.45 \\
\cline { 2 - 5 } & Hong Kong & 0.75 & 1.09 & 0.05 \\
\cline { 2 - 5 } & México & 1.56 & 2.04 & 0.44 \\
\hline
\end{tabular}

Fuente: Elaboración propia con base en información de los Bancos Centrales de los países en cuestión. Las tasas de interés reales (promedio mensual) se refieren a: Central Bank Prime Rate a 30 días (Brasil), Treasury Bonds a 30 días (Hong Kong) y Cetes a 28 días (México).

La tabla 6 muestra el coeficiente de correlación de las tasas de interés reales para cada par de países, en los 3 períodos de estudio. Es importante observar que durante el período de crisis de Hong Kong, éste es positivo entre los 3 mercados (contrario a la pre crisis). No obstante, en términos absolutos, la correlación entre Hong Kong y Brasil se incrementa de manera importante (indicando contagio) durante la crisis, mientras que ésta disminuye entre Hong Kong y México (descartando contagio).

En el período post crisis la correlación entre los mercados se vuelve nuevamente negativa. Esta cae entre Hong Kong y Brasil y se incrementa en los demás casos; la mayor correlación se da entre México y Brasil (efecto regional), aunque la poca información disponible en este período obliga a tomar estos resultados con cautela. 
TABla 6

\section{Comportamiento de los coeficientes de correlación de las tasas de interés reales de Hong Kong, Brasil y México en los períodos de pre crisis, crisis y post crisis en Hong Kong}

\begin{tabular}{|l|c|c|c|}
\hline & $\begin{array}{c}\text { Pre crisis } \\
10 / 96-09 / 97\end{array}$ & $\begin{array}{c}\text { Crisis } \\
10 / 97-01 / 98\end{array}$ & $\begin{array}{c}\text { Post crisis } \\
02 / 98-03 / 98\end{array}$ \\
\hline Hong Kong - Brasil & -0.19 & 0.66 & -0.35 \\
\hline Hong Kong - México & -0.17 & 0.05 & -0.31 \\
\hline México - Brasil & -0.22 & 0.36 & -0.52 \\
\hline
\end{tabular}

El coeficiente de correlación se estimó utilizando el procedimiento producto-momento de Pearson. Fuente: Elaboración propia basada en la tasa de interés real promedio mensual de, Central Bank Prime Rate a 30 días (Brasil), Treasury Bonds a 30 días (Hong Kong) y Cetes a 28 días (México).

\subsection{COMPORTAMIENTO DE LAS TASAS DE INTERÉS REALES DE ARGENTINA, BRASIL Y MÉXICO}

La tabla 7 muestra el valor de la media, el mínimo, el máximo y la desviación estándar de las tasas de interés reales de Argentina, Brasil y México, para los períodos antes, durante y después de la crisis argentina de 2001.

Puede observarse que durante esta crisis los valores medios de las tasas de interés reales aumentaron para Argentina y Brasil, pero cayeron para México. De nuevo, esto sugiere que la crisis argentina impactó con más fuerza a Brasil que a México. En efecto, México muestra una tendencia a la baja en sus tasas de interés reales, lo que indica que no fue contagiado por la crisis en Argentina.

En cuanto a Brasil, si bien pudiera haber cierto grado de contagio, éste se espera sea muy leve. Las tasas de interés reales aumentaron durante el período de crisis tan solo 3\%; la volatilidad de las mismas incluso disminuyó en $62 \%$.

En el período post crisis Argentina presenta tasas de interés reales negativas, con disminución en la volatilidad; en México y Brasil se observan tasas de 
EFECTOS DE LAS CRISIS ANTICIPADAS Y NO ANTICIPADAS SOBRE EL CONTAGIO FINANCIERO INTERNACIONAL

interés reales positivas, con incremento en la volatilidad. Esto muestra un comportamiento opuesto del mercado brasileño y mexicano respecto al argentino. Cabe mencionar que las tasas de interés reales son menores en la post crisis que en los demás períodos de estudio.

\section{TABLA 7}

\section{Comportamiento de las tasas de interés reales de Argentina, Brasil y México en los períodos de pre crisis, crisis y post crisis en Argentina (\%)}

\begin{tabular}{|l|l|c|c|c|}
\hline & & $\begin{array}{c}\text { Pre crisis } \\
10 / 99-10 / 01\end{array}$ & $\begin{array}{c}\text { Crisis } \\
11 / 01-08 / 02\end{array}$ & $\begin{array}{c}\text { Post crisis } \\
09 / 02-08 / 04\end{array}$ \\
\hline \multirow{3}{*}{ Media } & Argentina & 12.82 & 41.55 & -0.07 \\
\cline { 2 - 5 } & Brasil & 9.82 & 10.13 & 8.36 \\
\cline { 2 - 5 } & México & 6.50 & 3.05 & 2.28 \\
\hline \multirow{3}{*}{ Mánimo } & Argentina & 7.44 & 12.71 & -0.24 \\
\cline { 2 - 5 } & Brasil & 8.03 & 9.62 & 4.87 \\
\cline { 2 - 5 } & México & 3.19 & 2.04 & 0.89 \\
\hline \multirow{3}{*}{ D. estándar } & Argentina & 49.87 & 89.56 & -0.01 \\
\cline { 2 - 5 } & Brasil & 11.83 & 10.65 & 10.27 \\
\cline { 2 - 5 } & México & 10.30 & 4.01 & 4.15 \\
\cline { 2 - 5 } & Argentina & 9.04 & 27.43 & 0.08 \\
\cline { 2 - 5 } & Mrasil & 1.16 & 0.44 & 1.38 \\
\cline { 2 - 5 } & México & 1.95 & 0.61 & 1.01 \\
\hline
\end{tabular}

Fuente: Elaboración propia con base en información de los Bancos Centrales de los países en cuestión. Las tasas de interés reales (promedio mensual) se refieren a, Tasa de Referencia a 30 días (Argentina), Central Bank Prime Rate a 30 días (Brasil) y Cetes a 28 días (México).

La tabla 8 muestra el coeficiente de correlación de las tasas de interés reales para cada par de países, en los 3 períodos de estudio. Esta correlación aumenta en el período de crisis en todos los casos, y vuelve a caer en la post crisis. En el caso de Brasil-Argentina, el signo positivo del coeficiente podría sugerir contagio negativo, contrario a lo observado entre México-Argentina. 


\section{TABLA 8}

Comportamiento de los coeficientes de correlación de las tasas de interés reales de Argentina, Brasil y México en los períodos de pre crisis, crisis y post crisis en Argentina

\begin{tabular}{|l|c|c|c|}
\hline & $\begin{array}{c}\text { Pre crisis } \\
10 / 99-10 / 01\end{array}$ & $\begin{array}{c}\text { Crisis } \\
11 / 01-08 / 02\end{array}$ & $\begin{array}{c}\text { Post crisis } \\
09 / 02-08 / 04\end{array}$ \\
\hline Argentina - Brasil & 0.23 & 0.37 & -0.29 \\
\hline Argentina - México & -0.19 & -0.40 & -0.34 \\
\hline México - Brasil & -0.63 & -0.71 & 0.45 \\
\hline
\end{tabular}

El coeficiente de correlación se estimó utilizando el procedimiento producto-momento de Pearson. Fuente: Elaboración propia basada en la tasa de interés real promedio mensual de, Tasa de Referencia a 30 días (Argentina), Central Bank Prime Rate a 30 días (Brasil) y Cetes a 28 días (México).

\subsection{PRUEBA DE NORMALIDAD}

Las tablas 9 y 10 presentan el estadístico Jarque-Bera para la serie completa de los rendimientos del mercado accionario y de las tasas de interés reales, para cada país sujeto de estudio. En todos los casos se rechaza al $1 \%$ de significancia la condición de normalidad. Por ende, el modelo no será útil para realizar pronósticos (situación ya contemplada en este trabajo).

\section{RESULTADOS ECONOMÉTRICOS}

A continuación se presentan los resultados econométricos de la estimación del modelo desarrollado en la sección de metodología:

$$
\hat{e}_{b, t}=a_{b}+b_{b, t} \hat{e}_{a, t}+\mu_{b, t}
$$

con: 
TABLA 9

Pruebas de normalidad: Rendimiento accionario y tasas de interés reales $(10 / 96-03 / 98)$

\begin{tabular}{|c|c|c|}
\hline & $\begin{array}{c}\text { Rendimiento } \\
\text { accionario }\end{array}$ & $\begin{array}{c}\text { Tasas de interés } \\
\text { reales }\end{array}$ \\
\hline Brasil & 899.97 & 124.39 \\
& $(0.00)$ & $(0.00)$ \\
\hline Hong Kong & 2294.23 & 47.38 \\
& $(0.00)$ & $(0.00)$ \\
\hline México & 7669.14 & 45.33 \\
& $(0.00)$ & $(0.00)$ \\
\hline La prueba de normalidad se realiza utilizando el estadístico Jarque-Bera. \\
Entre paréntesis se encuentra el valor p.
\end{tabular}

Fuente: Elaboración propia con base en información de las Bolsas de Valores y Bancos Centrales de cada país en cuestión.

\section{TABLA 10}

Pruebas de normalidad: Rendimiento accionario $y$ tasas de interés reales $(10 / 99-08 / 04)$

\begin{tabular}{|c|c|c|}
\hline & $\begin{array}{l}\text { Rendimiento } \\
\text { accionario }\end{array}$ & $\begin{array}{c}\text { Tasas de interés } \\
\text { reales }\end{array}$ \\
\hline Argentina & $\begin{array}{l}715.11 \\
(0.00)\end{array}$ & $\begin{array}{l}195.00 \\
(0.00)\end{array}$ \\
\hline Brasil & $\begin{array}{l}17.11 \\
(0.00)\end{array}$ & $\begin{array}{l}22.43 \\
(0.00)\end{array}$ \\
\hline México & $\begin{array}{l}320.51 \\
(0.00)\end{array}$ & $\begin{array}{l}25.38 \\
(0.00)\end{array}$ \\
\hline
\end{tabular}

Fuente: Elaboración propia con base en información de las Bolsas de Valores y Bancos Centrales de cada país en cuestión. 


$$
\mathrm{b}_{\mathrm{b}, \mathrm{t}}=\mathrm{b}_{0}+\mathrm{b}_{1} \mathrm{D}_{1}
$$

En primera instancia se estima el modelo con mínimos cuadrados ordinarios. Se realizan las pruebas LM y White, con la finalidad de analizar la presencia de autocorrelación y heteroscedasticidad. Los resultados obtenidos muestran la existencia de ambos elementos (ver Anexo), por lo que el modelo se estima nuevamente con el procedimiento Newey-West.

Ante la posibilidad de que el contagio en los rendimientos accionarios y tasas de interés reales no se dé en el mismo período en que ocurre la crisis financiera, la ecuación (1) se amplía de la siguiente manera:

$$
\hat{\mathrm{e}}_{\mathrm{b}, \mathrm{t}+\mathrm{i}}=\mathrm{a}_{\mathrm{b}}+\mathrm{b}_{\mathrm{b}, \mathrm{t}} \hat{\mathrm{e}}_{\mathrm{a}, \mathrm{t}}+\mu_{\mathrm{b}, \mathrm{t}}, \text { con } \mathrm{i}=0,1, \ldots
$$

Para los casos de las crisis de Hong Kong en 1997 y de Argentina en 2001, el modelo de contagio de los rendimientos accionarios y tasas de interés reales se estima hasta con cuatro adelantos. Este rango ofrece un margen de tiempo suficiente para determinar el adelanto en que el coeficiente de crisis es significativo, hasta aquel en que deja de serlo.

\subsection{CRISIS DE HONG KONG: RENDIMIENTOS ACCIONARIOS}

La tabla 11 muestra que en el modelo sin adelantos, la relación entre los índices accionarios $\left(b_{b, t}\right)$ de Hong Kong con Brasil y de Hong Kong con México es positiva y significativa. Esto señala la dependencia diaria en el comportamiento de estos mercados bursátiles. Sin embargo, el coeficiente de contagio $\left(b_{1}\right)$ y la hipótesis de contagio $\left(\left|b_{1}\right|>\left|b_{0}\right|\right.$ y $\left.\left|b_{1}\right| \neq 0\right)$ no son significativos, de modo que no hay evidencia de contagio inmediato durante la crisis de Hong Kong.

De acuerdo a las tablas 11 y 12 , hay evidencia a favor de la hipótesis de contagio al transcurrir dos y tres días para el caso de Brasil (con el mayor efecto a los 3 días) y tres días para el caso de México. En estas tres ocasiones el coeficiente $b_{1}$ es positivo, lo que permite suponer la existencia de contagio negativo a raíz de la crisis de Hong Kong (siendo 
EFECTOS DE LAS CRISIS ANTICIPADAS Y NO ANTICIPADAS SOBRE EL CONTAGIO FINANCIERO INTERNACIONAL

el efecto ligeramente mayor en Brasil que en México). Esto es consistente con el análisis de la sección 4.1.

Todo lo anterior sugiere que al iniciarse la crisis en Hong Kong, la relación que mantenía con los mercados brasileño y mexicano era similar a la que prevalecía en períodos tranquilos. Al no prever la crisis, los inversionistas no ajustaron sus posiciones en América Latina de manera inmediata; sin embargo, al transcurrir pocos días, hubo un cambio en su percepción, de modo que ambos mercados se vieron afectados de manera directa por los movimientos accionarios ocurridos en Hong Kong.

\section{TABLA 11}

Resultados econométricos del modelo de contagio de la crisis de Hong Kong de 1997 sobre Brasil y México

(rendimientos accionarios)

\begin{tabular}{|c|c|c|c|c|c|c|c|c|c|c|}
\hline & \multicolumn{5}{|l|}{ Brasil } & \multicolumn{5}{|c|}{ México } \\
\hline & $a_{b}$ & $\mathrm{~b}_{\mathrm{b}, \mathrm{t}}$ & $\mathrm{b}_{0}$ & $\mathrm{~b}_{1}$ & $\mathrm{R}^{2}$ & $a_{b}$ & $\mathrm{~b}_{\mathrm{b}, \mathrm{t}}$ & $\mathrm{b}_{0}$ & $\mathrm{~b}_{1}$ & $\mathrm{R}^{2}$ \\
\hline $\begin{array}{l}0 \\
\text { adelantos }\end{array}$ & $\begin{array}{c}0.00 \\
(1.07)\end{array}$ & $\begin{array}{c}0.19 \\
(2.11)^{* *}\end{array}$ & $\begin{array}{c}0.15 \\
(1.54)\end{array}$ & $\begin{array}{c}0.07 \\
(0.39)\end{array}$ & 0.04 & $\begin{array}{c}0.00 \\
(0.99)\end{array}$ & $\begin{array}{c}0.13 \\
(1.95) * *\end{array}$ & $\begin{array}{c}0.18 \\
(4.18)^{* * * *}\end{array}$ & $\begin{array}{c}-0.07 \\
(-0.72)\end{array}$ & 0.05 \\
\hline $\begin{array}{l}1 \\
\text { adelanto }\end{array}$ & $\begin{array}{c}0.00 \\
(0.33)\end{array}$ & $\begin{array}{c}-0.05 \\
(-0.65)\end{array}$ & $\begin{array}{c}-0.01 \\
(-0.15)\end{array}$ & $\begin{array}{c}-0.06 \\
(-0.48)\end{array}$ & 0.00 & $\begin{array}{c}0.00 \\
(0.66)\end{array}$ & $\begin{array}{c}-0.05 \\
(-0.70)\end{array}$ & $\begin{array}{l}-0.05 \\
(1.06)\end{array}$ & $\begin{array}{c}-0.15 \\
(-1.54)\end{array}$ & 0.02 \\
\hline $\begin{array}{l}2 \\
\text { adelantos }\end{array}$ & $\begin{array}{c}0.00 \\
(1.67)^{*}\end{array}$ & $\begin{array}{c}0.20 \\
(1.50)\end{array}$ & $\begin{array}{c}-0.02 \\
(-0.34)\end{array}$ & $\begin{array}{c}0.35 \\
(1.91)^{*}\end{array}$ & 0.07 & $\begin{array}{c}0.00 \\
(0.91)\end{array}$ & $\begin{array}{c}0.16 \\
(1.69)^{*}\end{array}$ & $\begin{array}{c}0.04 \\
(1.12)\end{array}$ & $\begin{array}{c}0.20 \\
(1.39)\end{array}$ & 0.09 \\
\hline $\begin{array}{l}3 \\
\text { adelantos }\end{array}$ & $\begin{array}{c}0.00 \\
(1.21)\end{array}$ & $\begin{array}{c}0.13 \\
(2.10)^{* *}\end{array}$ & $\begin{array}{c}-0.03 \\
(-0.35)\end{array}$ & $\begin{array}{c}0.26 \\
(2.36)^{* *}\end{array}$ & 0.03 & $\begin{array}{c}0.00 \\
(0.89)\end{array}$ & $\begin{array}{c}0.06 \\
(1.54)\end{array}$ & $\begin{array}{l}-0.03 \\
(-0.75)\end{array}$ & $\begin{array}{c}0.13 \\
(1.99)^{* *}\end{array}$ & 0.02 \\
\hline $\begin{array}{l}4 \\
\text { adelantos }\end{array}$ & $\begin{array}{c}0.00 \\
(1.19)\end{array}$ & $\begin{array}{l}-0.05 \\
(-0.92)\end{array}$ & $\begin{array}{c}-0.06 \\
(-0.96)\end{array}$ & $\begin{array}{l}0.00 \\
(0.04)\end{array}$ & 0.00 & $\begin{array}{c}0.00 \\
(1.10)\end{array}$ & $\begin{array}{l}-0.05 \\
(-1.27)\end{array}$ & $\begin{array}{l}-0.05 \\
(-1.57)\end{array}$ & $\begin{array}{c}0.00 \\
(0.07)\end{array}$ & 0.00 \\
\hline
\end{tabular}

El modelo $\hat{\mathrm{e}}_{\mathrm{b}, \mathrm{t}+\mathrm{i}}=\mathrm{a}_{\mathrm{b}}+\mathrm{b}_{\mathrm{b}, \mathrm{t}} \hat{\mathrm{e}}_{\mathrm{a}, \mathrm{t}}+\mu_{\mathrm{b}, \mathrm{t}}$, con $\mathrm{i}=0, \ldots, 4$ se estimó mediante mínimos cuadrados ordinarios, utilizando el método Newey-West. Se toma $b_{b, t}=b_{0}+b_{1} D_{1}$. 
TABLA 12

Prueba Wald: Estadístico F para los coeficientes estimados de la ecuación (3), durante la crisis de Hong Kong de 1997

\begin{tabular}{|l|l|l|l|}
\hline $\begin{array}{l}\text { Hipótesis } \\
\left(\mathrm{H}_{0}\right)\end{array}$ & $\begin{array}{l}\text { Brasil } \\
2 \text { adelantos }\end{array}$ & $\begin{array}{l}\text { Brasil } \\
3 \text { adelantos }\end{array}$ & $\begin{array}{l}\text { México } \\
3 \text { adelantos }\end{array}$ \\
\hline $\mathrm{b}_{\mathrm{b}, \mathrm{t}}=0$ & 2.27 & $6.65^{* *}$ & 2.40 \\
\hline$\left|\mathrm{b}_{0}\right|=\left|\mathrm{b}_{1}\right|$ & $3.54^{*}$ & $10.34^{* *}$ & $3.63^{*}$ \\
\hline $\mathrm{b}_{0}=0$ & 0.11 & 0.12 & 0.56 \\
\hline $\begin{array}{l}\mathrm{b}_{1}=0 \\
\text { * Se rechaza } \mathrm{H}_{0} \text { al } 10 \% \text { de significancia } \\
\text { ** Se rechaza } \mathrm{H}_{0} \text { al 5\% de significancia } \\
\text { *** Se rechaza } \mathrm{H}_{0} \text { al } 1 \% \text { de significancia }\end{array}$ & $3.97^{* * *}$ \\
\hline
\end{tabular}

\subsection{CRISIS DE ARGENTINA: RENDIMIENTOS ACCIONARIOS}

Al estimar el modelo sin adelantos, se puede apreciar en la tabla 13 que la relación entre los índices accionarios $\left(\mathrm{b}_{\mathrm{b}, \mathrm{t}}\right.$ ) de Argentina con Brasil y de Argentina con México es positiva y significativa. Tanto los coeficientes de los períodos tranquilo y de crisis son significativos, pero de signo opuesto. Esto sugiere, en un primer momento, la posible existencia de un contagio positivo e inmediato de la crisis argentina.

De acuerdo a las tablas 13 y 14, la hipótesis de contagio $\left(\left|b_{1}\right|>\left|b_{0}\right|\right.$ y $\left.\left|b_{1}\right| \neq 0\right)$ no es significativa en ningún adelanto. Por lo tanto, no se puede suponer la existencia de contagio de parte del mercado argentino. Esto es consistente con el análisis de la sección 4.4. Esta situación evidencia la anticipación de la crisis argentina por parte de los inversionistas quienes, al no ser sorprendidos por la misma, frenaron el efecto contagio. 
EFECTOS DE LAS CRISIS ANTICIPADAS Y NO ANTICIPADAS SOBRE EL CONTAGIO FINANCIERO INTERNACIONAL

TABLA 13

Resultados econométricos del modelo de contagio de la crisis argentina de 2001 sobre Brasil y México

(rendimientos accionarios)

\begin{tabular}{|c|c|c|c|c|c|c|c|c|c|c|}
\hline & \multicolumn{5}{|l|}{ Brasil } & \multicolumn{5}{|c|}{ México } \\
\hline & $a_{b}$ & $\mathrm{~b}_{\mathrm{b}, \mathrm{t}}$ & $\mathrm{b}_{0}$ & $\mathrm{~b}_{1}$ & $\mathrm{R}^{2}$ & $a_{b}$ & $b_{b, t}$ & $\mathrm{~b}_{0}$ & $\mathrm{~b}_{1}$ & $\mathrm{R}^{2}$ \\
\hline $\begin{array}{l}0 \\
\text { adelantos }\end{array}$ & $\begin{array}{c}0.00 \\
(1.07)\end{array}$ & $\begin{array}{c}0.25 \\
(6.23)^{* * *}\end{array}$ & $\begin{array}{c}0.39 \\
(9.95)^{* * *}\end{array}$ & $\begin{array}{c}-0.35 \\
(-5.56)^{* * *}\end{array}$ & 0.15 & $\begin{array}{c}0.00 \\
(1.09)\end{array}$ & $\begin{array}{c}0.17 \\
(6.63)^{* * *}\end{array}$ & $\begin{array}{c}0.26 \\
(7.99) * *\end{array}$ & $\begin{array}{c}-0.21 \\
(-5.69)^{* *}\end{array}$ & 0.11 \\
\hline $\begin{array}{l}1 \\
\text { adelanto }\end{array}$ & $\begin{array}{c}0.00 \\
(1.14)\end{array}$ & $\begin{array}{c}-0.02 \\
(-0.71)\end{array}$ & $\begin{array}{c}-0.01 \\
(-0.31)\end{array}$ & $\begin{array}{c}-0.02 \\
(-0.38)\end{array}$ & 0.00 & $\begin{array}{c}0.00 \\
(1.18)\end{array}$ & $\begin{array}{c}0.02 \\
(1.37)\end{array}$ & $\begin{array}{c}0.04 \\
(1.89)^{*}\end{array}$ & $\begin{array}{c}-0.04 \\
(-1.34)\end{array}$ & 0.00 \\
\hline $\begin{array}{l}2 \\
\text { adelantos }\end{array}$ & $\begin{array}{c}0.00 \\
(0.85)\end{array}$ & $\begin{array}{c}-0.03 \\
(-1.13)\end{array}$ & $\begin{array}{c}0.01 \\
(0.23)\end{array}$ & $\begin{array}{c}-0.02 \\
(-0.62)\end{array}$ & 0.00 & $\begin{array}{c}0.00 \\
(0.90)\end{array}$ & $\begin{array}{c}0.00 \\
(0.17)\end{array}$ & $\begin{array}{c}0.00 \\
(0.21)\end{array}$ & $\begin{array}{c}-0.01 \\
(-0.16)\end{array}$ & 0.00 \\
\hline $\begin{array}{l}3 \\
\text { adelantos }\end{array}$ & $\begin{array}{c}0.00 \\
(0.55)\end{array}$ & $\begin{array}{c}0.00 \\
(0.53)\end{array}$ & $\begin{array}{c}0.03 \\
(0.80)\end{array}$ & $\begin{array}{c}-0.07 \\
(-1.32)\end{array}$ & 0.00 & $\begin{array}{c}0.00 \\
(0.96)\end{array}$ & $\begin{array}{c}0.00 \\
(0.95)\end{array}$ & $\begin{array}{c}0.02 \\
(0.93)\end{array}$ & $\begin{array}{c}-0.03 \\
(-0.26)\end{array}$ & 0.00 \\
\hline $\begin{array}{l}4 \\
\text { adelantos }\end{array}$ & $\begin{array}{c}0.00 \\
(1.19)\end{array}$ & $\begin{array}{c}0.00 \\
(1.18)\end{array}$ & $\begin{array}{c}0.04 \\
(1.40)\end{array}$ & $\begin{array}{c}-0.03 \\
(-0.27)\end{array}$ & 0.00 & $\begin{array}{c}0.00 \\
(1.21)\end{array}$ & $\begin{array}{c}0.00 \\
(1.20)\end{array}$ & $\begin{array}{c}0.04 \\
(1.75)^{*}\end{array}$ & $\begin{array}{c}-0.04 \\
(-1.14)\end{array}$ & 0.00 \\
\hline
\end{tabular}

El modelo $\hat{\mathrm{e}}_{\mathrm{b}, \mathrm{t}+\mathrm{i}}=\mathrm{a}_{\mathrm{b}}+\mathrm{b}_{\mathrm{b}, \mathrm{t}} \hat{\mathrm{e}}_{\mathrm{a}, \mathrm{t}}+\mu_{\mathrm{b}, \mathrm{t}}$, con $\mathrm{i}=0, \ldots, 4$ se estimó mediante mínimos cuadrados ordinarios, utilizando el método Newey-West. Se toma $b_{b, t}=b_{0}+b_{1} D_{1}$.

\section{Tabla 14}

Prueba Wald: Estadístico F para los coeficientes estimados de la ecuación (3), durante la crisis de Argentina de 2001

\begin{tabular}{|l|l|l|}
\hline Hipótesis $\left(\mathrm{H}_{\mathrm{O}}\right)$ & $\begin{array}{l}\text { Brasil } \\
0 \text { adelantos }\end{array}$ & $\begin{array}{l}\text { México } \\
0 \text { adelantos }\end{array}$ \\
\hline \multicolumn{2}{|l|}{$\mathrm{b}_{\mathrm{b}, \mathrm{t}}=0$} & $38.83 * *$ \\
\hline$\left|\mathrm{b}_{0}\right|=\mid \mathrm{b}_{1}$ & 0.71 & $44.09 * *$ \\
\hline $\mathrm{b}_{0}=0$ & $99.14 * *$ & $63.97 * *$ \\
\hline $\mathrm{b}_{1}=0$ & $30.95 * *$ & $32.41 * *$ \\
\hline $\begin{array}{l}* \text { Se rechaza } \mathrm{H}_{0} \text { al } 5 \% \text { de significancia } \\
* * \text { Se rechaza } \mathrm{H}_{0} \text { al } 1 \% \text { de significancia }\end{array}$ \\
\hline
\end{tabular}




\subsection{CRISIS DE HONG KONG: TASAS DE INTERÉS REALES}

La tabla 15 muestra que en el modelo sin adelantos, la relación entre la tasa de interés real $\left(b_{b, t}\right)$ entre Hong Kong y Brasil así como entre Hong Kong y México es positiva y significativa. Aunado a que el coeficiente $\mathrm{b}_{0}$ es positivo y significativo, hay indicios que en tiempos normales las tasas de interés reales de estos países se mueven en la misma dirección, de manera inmediata. No obstante, el coeficiente de contagio $\left(b_{1}\right)$ y la hipótesis de contagio $\left(\left|b_{1}\right|>\left|b_{0}\right| y\left|b_{1}\right| \neq 0\right)$ no son significativos en ninguno de los dos casos, por lo que no hay evidencia de contagio inmediato de la crisis de Hong Kong. Esto refleja la falta de anticipación de esta crisis.

De acuerdo a las tablas 15 y 16, la hipótesis de contagio (en este caso negativo) es significativa para Brasil después de transcurrido un mes (aunque con una diferencia pequeña entre los coeficientes $b_{0} y_{b_{1}}$ ). Se observa un vínculo entre las tasas de interés de Brasil y Hong Kong hasta con tres adelantos; sin embargo, la mayor relación se percibe con un solo adelanto.

La mayor relación entre las tasas de interés de Hong Kong y México se da en el modelo sin adelantos. Sin embargo, para México la hipótesis de contagio se rechaza en todos los casos.

Lo anterior es consistente con los resultados expuestos en secciones anteriores. Resumiendo, la crisis en Hong Kong tuvo un mayor impacto sobre Brasil que sobre México. Este efecto negativo de contagio no fue inmediato, lo que descarta la anticipación de la crisis de Hong Kong.

\subsection{CRISIS DE ARGENTINA: TASAS DE INTERÉS REALES}

Los resultados del modelo sin adelantos que se observan en la tabla 17, muestran que la relación entre la tasa de interés real $\left(\mathrm{b}_{\mathrm{b}, \mathrm{t}}\right)$ de Argentina con Brasil y de Argentina con México es directa y significativa. Para ambos países el coeficiente del período tranquilo es significativo y positivo; sin embargo, el coeficiente de crisis (con valor negativo) solamente es significativo para México, lo cual en una primera aproximación supone la existencia de contagio positivo inmediato. 
EFECTOS DE LAS CRISIS ANTICIPADAS Y NO ANTICIPADAS SOBRE EL CONTAGIO FINANCIERO INTERNACIONAL

TABLA 15

Resultados econométricos del modelo de contagio de la crisis de Hong Kong de 1997 sobre Brasil y México

(tasas de interés reales)

\begin{tabular}{|c|c|c|c|c|c|c|c|c|c|c|}
\hline & \multicolumn{5}{|l|}{ Brasil } & \multicolumn{5}{|c|}{ México } \\
\hline & $a_{b}$ & $\mathrm{~b}_{\mathrm{b}, \mathrm{t}}$ & $\mathrm{b}_{0}$ & $\mathrm{~b}_{1}$ & $\mathrm{R}^{2}$ & $a_{b}$ & $\mathrm{~b}_{\mathrm{b}, \mathrm{t}}$ & $\mathrm{b}_{0}$ & $b_{1}$ & $\mathrm{R}^{2}$ \\
\hline $\begin{array}{l}0 \\
\text { adelantos }\end{array}$ & $\begin{array}{c}0.15 \\
(0.62)\end{array}$ & $\begin{array}{c}3.52 \\
(3.11)^{* * * *}\end{array}$ & $\begin{array}{c}3.73 \\
(2.44)^{* * * *}\end{array}$ & $\begin{array}{c}-0.30 \\
(-0.16)\end{array}$ & 0.49 & $\begin{array}{c}0.01 \\
(0.46)\end{array}$ & $\begin{array}{c}0.43 \\
(4.63)^{* * * *}\end{array}$ & $\begin{array}{c}0.63 \\
(1.89)^{*}\end{array}$ & $\begin{array}{l}-0.31 \\
(-0.95)\end{array}$ & 0.25 \\
\hline $\begin{array}{l}1 \\
\text { adelanto }\end{array}$ & $\begin{array}{c}0.14 \\
(0.43)\end{array}$ & $\begin{array}{c}4.23 \\
(3.75)^{* * *}\end{array}$ & $\begin{array}{c}2.68 \\
(2.56)^{* * *}\end{array}$ & $\begin{array}{c}2.70 \\
(2.14)^{* * *}\end{array}$ & 0.88 & $\begin{array}{l}0.01 \\
(0.10)\end{array}$ & $\begin{array}{c}0.15 \\
(15.54)^{* * * *}\end{array}$ & $\begin{array}{c}0.85 \\
(4.04)^{* * *}\end{array}$ & $\begin{array}{c}-0.56 \\
(-2.49) * *\end{array}$ & 0.36 \\
\hline $\begin{array}{l}2 \\
\text { adelantos }\end{array}$ & $\begin{array}{c}0.16 \\
(0.72)\end{array}$ & $\begin{array}{c}3.14 \\
(4.68) * *\end{array}$ & $\begin{array}{c}4.26 \\
(2.28)^{* * *}\end{array}$ & $\begin{array}{c}-0.40 \\
(-0.17)\end{array}$ & 0.69 & $\begin{array}{c}0.01 \\
(0.83)\end{array}$ & $\begin{array}{c}0.16 \\
(16.59)^{* * *}\end{array}$ & $\begin{array}{c}1.10 \\
(4.79) * * * *\end{array}$ & $\begin{array}{c}-0.85 \\
(-3.60) * * *\end{array}$ & 0.52 \\
\hline $\begin{array}{l}3 \\
\text { adelantos }\end{array}$ & $\begin{array}{c}0.19 \\
(0.42)\end{array}$ & $\begin{array}{c}1.09 \\
(3.27)^{* * *}\end{array}$ & $\begin{array}{c}6.62 \\
(2.64)^{* * *}\end{array}$ & $\begin{array}{c}-4.49 \\
(-1.49)\end{array}$ & 0.64 & $\begin{array}{c}0.00 \\
(0.45)\end{array}$ & $\begin{array}{c}0.22 \\
(14.38)^{* * *}\end{array}$ & $\begin{array}{c}0.91 \\
(3.66)^{* * * *}\end{array}$ & $\begin{array}{c}-0.74 \\
(-3.06) * * *\end{array}$ & 0.43 \\
\hline $\begin{array}{l}4 \\
\text { adelantos }\end{array}$ & $\begin{array}{c}0.20 \\
(0.44)\end{array}$ & $\begin{array}{c}3.02 \\
(0.25)\end{array}$ & $\begin{array}{c}6.80 \\
(0.43)\end{array}$ & $\begin{array}{c}-5.30 \\
(-0.48)\end{array}$ & 0.56 & $\begin{array}{c}0.00 \\
(0.83)\end{array}$ & $\begin{array}{c}0.16 \\
(16.59)^{* * * *}\end{array}$ & $\begin{array}{c}0.56 \\
(2.49)^{* * *}\end{array}$ & $\begin{array}{c}-0.39 \\
(-1.66)\end{array}$ & 0.52 \\
\hline \multicolumn{11}{|c|}{$\begin{array}{l}* \text { significativo al } 10 \% \\
* * \text { significativo al } 5 \% \\
* * * \text { significativo al } 1 \% \\
\text { Lo que está entre parén }\end{array}$} \\
\hline
\end{tabular}

El modelo $\hat{\mathrm{e}}_{\mathrm{b}, \mathrm{t}+\mathrm{i}}=\mathrm{a}_{\mathrm{b}}+\mathrm{b}_{\mathrm{b}, \mathrm{t}} \hat{\mathrm{e}}_{\mathrm{a}, \mathrm{t}}+\mu_{\mathrm{b}, \mathrm{t}}$, con $\mathrm{i}=0, \ldots, 4$ se estimó mediante mínimos cuadrados ordinarios, utilizando el método Newey-West. Se toma $b_{b, t}=b_{0}+b_{1} D_{1}$.

\section{TABLA 16}

Prueba Wald: Estadístico F para los coeficientes estimados de la ecuación (3), durante la crisis de Hong Kong de 1997

\begin{tabular}{|c|c|c|c|c|}
\hline $\begin{array}{l}\text { Hipótesis } \\
\left(\mathrm{H}_{0}\right)\end{array}$ & $\begin{array}{l}\text { Brasil } \\
1 \text { adelanto }\end{array}$ & $\begin{array}{l}\text { México } \\
1 \text { adelanto }\end{array}$ & $\begin{array}{l}\text { México } \\
2 \text { adelantos }\end{array}$ & $\begin{array}{l}\text { México } \\
3 \text { adelantos }\end{array}$ \\
\hline $\mathrm{b}_{\mathrm{b}, \mathrm{t}}=0$ & $14.10^{*}$ & $53.55 * *$ & $88.27^{* *}$ & $60.15^{* *}$ \\
\hline$\left|b_{0}=\right| b_{1} \mid$ & $6.14 *$ & $11.27 * *$ & $18.19 * *$ & $7.51 *$ \\
\hline $\mathrm{b}_{0}=0$ & $6.55^{*}$ & $16.33 * *$ & $23.02 * *$ & $13.42 * *$ \\
\hline $\mathrm{b}_{1}=0$ & $6.33 *$ & $6.20 *$ & $12.99 * *$ & $9.40 * *$ \\
\hline
\end{tabular}


De acuerdo a las tablas 17 y 18, tanto para México como para Brasil, la hipótesis de contagio $\left(\left|b_{1}\right|>\left|b_{0}\right|\right.$ y $\left.\left|b_{1}\right| \neq 0\right)$ no es significativa en ningún momento, lo que no permite suponer la existencia de contagio de la crisis argentina. Esto es consistente con los resultados de las secciones anteriores.

Resumiendo, la crisis argentina no contagió ni a Brasil ni a México, lo cual confirma que fue una crisis anticipada.

\section{TABLA 17}

\section{Resultados econométricos del modelo de contagio de la crisis argentina de 2001 sobre Brasil y México (tasas de interés reales)}

\begin{tabular}{|c|c|c|c|c|c|c|c|c|c|c|}
\hline & \multicolumn{5}{|l|}{ Brasil } & \multicolumn{5}{|c|}{ México } \\
\hline & $a_{b}$ & $\mathrm{~b}_{\mathrm{b}, \mathrm{t}}$ & $\mathrm{b}_{0}$ & $\mathrm{~b}_{1}$ & $\mathrm{R}^{2}$ & $a_{b}$ & $b_{b, t}$ & $\mathrm{~b}_{0}$ & $\mathrm{~b}_{1}$ & $\mathrm{R}^{2}$ \\
\hline $\begin{array}{l}0 \\
\text { adelantos }\end{array}$ & $\begin{array}{c}0.09 \\
(0.70)\end{array}$ & $\begin{array}{c}0.12 \\
(19.64)^{* * * *}\end{array}$ & $\begin{array}{c}0.39 \\
(2.18)^{* *}\end{array}$ & $\begin{array}{c}-0.02 \\
(-1.04)\end{array}$ & 0.18 & $\begin{array}{c}0.04 \\
(0.47)\end{array}$ & $\begin{array}{c}0.02 \\
(2.13)^{* * *}\end{array}$ & $\begin{array}{c}0.09 \\
(2.37)^{* *}\end{array}$ & $\begin{array}{c}-0.11 \\
(-2.59)^{* *}\end{array}$ & 0.27 \\
\hline $\begin{array}{l}1 \\
\text { adelanto }\end{array}$ & $\begin{array}{c}0.08 \\
(0.39)\end{array}$ & $\begin{array}{c}0.02 \\
(22.83)^{* * *}\end{array}$ & $\begin{array}{c}0.05 \\
(2.64)^{* *}\end{array}$ & $\begin{array}{c}-0.03 \\
(-1.59)\end{array}$ & 0.24 & $\begin{array}{c}0.04 \\
(0.58)\end{array}$ & $\begin{array}{c}-0.03 \\
(2.49)^{* * *}\end{array}$ & $\begin{array}{c}0.09 \\
(1.97)^{*}\end{array}$ & $\begin{array}{c}-0.10 \\
(-2.18)^{* *}\end{array}$ & 0.23 \\
\hline $\begin{array}{l}2 \\
\text { adelantos }\end{array}$ & $\begin{array}{c}0.09 \\
(0.88)\end{array}$ & $\begin{array}{c}0.01 \\
(28.72)^{* * * *}\end{array}$ & $\begin{array}{c}0.05 \\
(3.28)^{* *}\end{array}$ & $\begin{array}{c}-0.03 \\
(-2.17)^{* *}\end{array}$ & 0.27 & $\begin{array}{c}0.05 \\
(1.02)\end{array}$ & $\begin{array}{c}-0.01 \\
(2.23)^{* *}\end{array}$ & $\begin{array}{c}0.09 \\
(2.17)^{* *}\end{array}$ & $\begin{array}{c}.-0.11 \\
(-2.30)^{* *}\end{array}$ & 0.23 \\
\hline $\begin{array}{l}3 \\
\text { adelantos }\end{array}$ & $\begin{array}{c}0.08 \\
(0.75)\end{array}$ & $\begin{array}{c}0.04 \\
(1.02)\end{array}$ & $\begin{array}{c}0.00 \\
(0.83)\end{array}$ & $\begin{array}{c}0.07 \\
(1.06)\end{array}$ & 0.12 & $\begin{array}{c}0.01 \\
(0.70)\end{array}$ & $\begin{array}{c}-0.12 \\
(-1.31)\end{array}$ & $\begin{array}{l}-0.46 \\
(-1.31)\end{array}$ & $\begin{array}{c}0.12 \\
(0.89)\end{array}$ & 0.22 \\
\hline $\begin{array}{l}4 \\
\text { adelantos }\end{array}$ & $\begin{array}{c}0.09 \\
(0.66)\end{array}$ & $\begin{array}{c}0.03 \\
(0.04)\end{array}$ & $\begin{array}{c}0.06 \\
(0.75)\end{array}$ & $\begin{array}{c}-0.05 \\
(-0.62)\end{array}$ & 0.08 & $\begin{array}{c}0.04 \\
(0.94)\end{array}$ & $\begin{array}{c}-0.04 \\
(-1.05)\end{array}$ & $\begin{array}{c}0.09 \\
(0.94)\end{array}$ & $\begin{array}{c}-0.11 \\
(-0.84)\end{array}$ & 0.21 \\
\hline \multicolumn{11}{|c|}{$\begin{array}{l}* \text { significativo al } 10 \% \\
* * \text { significativo al } 5 \% \\
* * * \text { significativo al } 1 \% \\
\text { Lo que está entre parént }\end{array}$} \\
\hline
\end{tabular}

El modelo $\hat{\mathrm{e}}_{\mathrm{b}, \mathrm{t}+\mathrm{i}}=\mathrm{a}_{\mathrm{b}}+\mathrm{b}_{\mathrm{b}, \mathrm{t}} \hat{\mathrm{e}}_{\mathrm{a}, \mathrm{t}}+\mu_{\mathrm{b}, \mathrm{t}}$, con $\mathrm{i}=0, \ldots, 4$ se estimó mediante mínimos cuadrados ordinarios, utilizando el método Newey-West. Se toma $b_{b, t}=b_{0}+b_{1} D_{1}$. 
EFECTOS DE LAS CRISIS ANTICIPADAS Y NO ANTICIPADAS SOBRE EL CONTAGIO FINANCIERO INTERNACIONAL

Tabla 18

Prueba Wald: Estadístico F para los coeficientes estimados de la ecuación (3), durante la crisis de Argentina de 2001

\begin{tabular}{|c|c|c|c|c|}
\hline $\begin{array}{l}\text { Hipótesis } \\
\left(\mathrm{H}_{0}\right)\end{array}$ & $\begin{array}{l}\text { Brasil } \\
2 \text { adelantos }\end{array}$ & $\begin{array}{l}\text { México } \\
0 \text { adelantos }\end{array}$ & $\begin{array}{l}\text { México } \\
1 \text { adelanto }\end{array}$ & $\begin{array}{l}\text { México } \\
2 \text { adelantos }\end{array}$ \\
\hline$b_{b, t}=0$ & $13.03 * * *$ & $4.18 *$ & $6.47 * *$ & $5.32 * *$ \\
\hline$\left|\mathrm{b}_{0}\right|=\mid \mathrm{b}_{1}$ & $13.95 * * *$ & 2.27 & 2.24 & 1.45 \\
\hline $\mathrm{b}_{0}=0$ & $10.78 * * *$ & $5.64 * *$ & $3.90 *$ & $4.73 * *$ \\
\hline$b_{1}=0$ & $4.73 * *$ & $6.71 * *$ & $4.77 * *$ & $5.31 * *$ \\
\hline \multicolumn{5}{|c|}{$\begin{array}{l}\text { * Se rechaza } \mathrm{H}_{0} \text { al } 10 \% \text { de significancia } \\
* * \text { Se rechaza } \mathrm{H}_{0} \text { al } 5 \% \text { de significancia } \\
* * \text { Se rechaza } \mathrm{H}_{0} \text { al } 1 \% \text { de significancia }\end{array}$} \\
\hline
\end{tabular}

\section{CONCLUSIONES Y LIMITACIONES}

La literatura sobre crisis financieras explica que la anticipación de una crisis por parte de los inversionistas evita que se dé el efecto contagio. Este artículo presenta evidencia a favor de este argumento, donde se define el contagio como un aumento significativo en la correlación entre las variables económicas y/o financieras de dos ó más países, inducido por un choque externo.

En este estudio se analizan los efectos contagio de dos crisis financieras recientes sobre Brasil y México: la crisis argentina (considerada como anticipada) en el año 2001 y la ocurrida en Hong Kong (considerada como no anticipada) durante 1997. Se escogen Brasil y México por ser dos de las economías más grandes y abiertas de la región de América Latina. Como variables de análisis se toman los rendimientos accionarios y las tasas de interés reales.

Los resultados muestran que la crisis financiera de Hong Kong ocasionó un evento de contagio sobre los rendimientos accionarios tanto de Brasil como de México, siendo el efecto mayor sobre Brasil. Al analizar el comportamiento de las tasas de interés reales, el contagio se da únicamente hacia 
Brasil. Se comprueba que la crisis de Hong Kong no fue anticipada por los inversionistas, debido a que el contagio no se presenta de forma inmediata, sino que aparece en periodos posteriores al inicio de la crisis.

Durante el período de crisis de Hong Kong, éste no posee relaciones comerciales o financieras fuertes con Brasil ni México. Se observa que en el mes que inicia la crisis, las exportaciones de México y de Brasil hacia Hong Kong representan respectivamente solo el $0.26 \%$ y el $0.35 \%$ de sus exportaciones totales (INEGI, 1998; IBGE, 1998). El hecho de que el efecto de la crisis de Hong Kong sea más significativo sobre el mercado brasileño que sobre el mexicano, puede explicarse más bien por los fundamentos económicos y financieros de los países seleccionados. Brasil presentaba ciertas características que lo hacían vulnerable a experimentar un ataque especulativo, con repercusiones en el mercado accionario y tasas de interés: tipo de cambio fijo (el cual mostraba ya debilidades; en el primer trimestre de 1997 el Banco Central de Brasil utilizó 7.5 billones de dólares para defender la paridad), déficit fiscal y de cuenta corriente considerables. Por otra parte, si bien la crisis mexicana de 1994 era reciente, los agentes no tenían incentivos para realizar un ataque especulativo. Desde el año 1995 el tipo de cambio había dejado de ser fijo (se consideraba incluso que la moneda estaba subvaluada) y la economía presentaba un saldo positivo de balanza comercial (Dornbusch y Cline, 1997; Evangelist y Sathe, 2006).

Para el caso de la crisis argentina de 2001, no hay evidencia de contagio sobre los rendimientos accionarios o tasas de interés reales de Brasil ni México. Es un resultado interesante, especialmente al considerar la gran vinculación comercial y financiera entre estos países. Lo anterior muestra que esta crisis ya era anticipada por los inversionistas.

Resumiendo, la crisis financiera (no anticipada) de Hong Kong sí ocasionó contagio especialmente sobre Brasil, mientras que la crisis ocurrida en Argentina (anticipada) no tuvo ningún efecto sobre los países de estudio. Si bien el propósito de este artículo consiste únicamente en analizar si hubo un incremento en la correlación normal existente entre los rendimientos accionarios y las tasas de interés reales de los mercados seleccionados, una limitación del 
EFECTOS DE LAS CRISIS ANTICIPADAS Y NO ANTICIPADAS SOBRE EL CONTAGIO FINANCIERO INTERNACIONAL

estudio es la falta de un análisis estructural. Para ello, habría que incluir otras variables que pudieran cambiar o confirmar los resultados obtenidos a la fecha; algunas de estas variables son: reservas monetarias internacionales, base monetaria e índices de confianza del consumidor y del productor. No obstante, la periodicidad de estos datos es baja (mensual en la mayoría de los casos), por lo que no sería posible estudiar el efecto contagio inmediato (es decir, con pocos días de adelanto).

\section{BIBLIOGRAFÍA}

Alba, P., A. Bhattacharya, S. Claessens, S. Ghosh y L. Hernandez (1998), "Volatility and Contagion in a Financially Integrated World: Lessons from East Asia's Recent Experience", Banco Mundial y Banco Central de Chile, documento de trabajo, núm 2008.

Baele, L. y K. Inghelbrecht (2006), “Time Varying-Integration Interdependence and Contagion", Tilburg University y Ghent University, documento de trabajo, versión preliminar.

Baig, T. y I. Goldfjan (2000), "The Russian Default and the Contagion to Brazil", Fondo Monetario Internacional, documento de trabajo, núm. 160.

Banco Central de la República Argentina (2007), Estadísticas e Indicadores; recuperado de <www.bcra.gov.ar>.

Banxico (1997), Estadísticas Económicas y Financieras, recuperado de <www.banxico.org.mx>.

Bayoumi, T., G. Fazio, M. Kumar y R. MacDonald (2003), "Fatal Attraction: A New Measure of Contagion", Fondo Monetario Internacional, documento de trabajo, núm. 80.

Bekaert, G., H. Campbell y A. Ng (2005), "Market Integration and Contagion", Journal of Business, Vol. 78, núm. 1, pp. 39-70.

Bikhchandani, S. (1992), “A Bargaining Model with Incomplete Information”, Review of Economics Studies, Vol. 59, núm. 1, pp.187-203.

Boschi, M. (2005), "International Financial Contagion: Evidence from the Argentina Crisis of 2001-2002”, Applied Financial Economics, Vol. 15, núm. 3, pp. 153-163. 
Bustelo, P. (2007), "Capital Flows and Financial Crises: A Comparative Analysis of East Asia (1997-1998) and Argentina (2001-2002)”, en: K. N. Rao (comp.), Exchange Rate Management in Emerging Economies of Asia, ICFAI University Press, Hyderabad.

Calvo, G. (2006), "Monetary Policy Challenges in Emerging Markets: Sudden Stop, Liability Dollarization, and Lender of Last Resort", Banco Interamericano de Desarrollo, documento de trabajo, núm. 596.

Calvo, G. (2005), "Crises in Emerging Market Economies. A Global Perspective", National Bureau of Economic Research, documento de trabajo, núm. 11305.

Calvo, S. y C. Reinhart (1996), "Capital Inflows to Latin America: Is there Evidence of Contagion Effects?”, World Bank Policy Research, documento de trabajo, núm. 1619.

Calvo, G. y E. Mendoza (1999), "Regional Contagion and the Globalization of Securities Markets", National Bureau of Economic Research, documento de trabajo, núm. 7153.

Caramazza, F., L. Ricci y R. Salgado (2004), "International Financial Contagion in Currency Crises”, Journal of International Money and Finance, Vol. 23, núm.1, pp. 51-70.

Chen, F. y R. Ng (2000), "Regaining International Competitiveness: Hong Kong after the Asian Financial Crisis”, en: S. Masuyama, D. Vandenbrink y Chia Siow Yue (eds), Restoring East Asia's Dynamism, Tokyo, Nomura Research Institute y Singapore Institute of Southeast Asian Studies, pp. 134-162.

Chul Park, Y. y C. Song (1999), "Financial Contagion in the East Asian Crisis", Korea University, documento de trabajo.

Corsetti, G., P. Pesenti y N. Roubini (1999), "What Caused the Asian Currency and Financial Crises?", Japan and the World Economy, Vol. 11, núm. 3, pp. 305-373.

Daseking, C., A. Ghosh, T. Lane y A. Thomas (2005), "Lessons from the Crisis in Argentina", Fondo Monetario Internacional, ISBN-10: 1589063597.

De la Torre, A., E. Yeyati y S. Schumukler (2002), “Argentina's Financial Crisis: Floating Money, Sinking Banking”, Banco Mundial, Mimeo. 
EFECTOS DE LAS CRISIS ANTICIPADAS Y NO ANTICIPADAS SOBRE EL CONTAGIO FINANCIERO INTERNACIONAL

Didier, T., P. Mauro y S. Schmukler (2006), “Vanishing Contagion?”, Fondo Monetario Internacional, Policy Discussion Paper, Research Department, núm. 01.

Dornbusch, R. y W. Cline (1997), "Brazil’s Incomplete Stabilization and Reform”, Brookings Papers on Economic Activity, Vol. 1997, núm. 1, pp. 367-404.

Dornbusch, R., Y. Chul Park. y S. Claessens (2000), "Contagion: Understanding how it Spreads", The World Bank Research Observer, Vol. 15, núm. 2, pp. 177-197.

Dungey, M., R. Fry, B. Gonzalez y V. Martin (2005), “A Comparison of Alternative Tests of Contagion with Applications”, en: M. Dungey y D. Tambakis (eds), International Financial Contagion: A Reader, Oxford University Press.

Edwards, S. y R. Susmel (1999), “Contagion and Volatility in the 1990’'s”, Universidad del Cema, documento de trabajo, núm. 153.

Eichengreen, B., A. Rose y C. Wyplosz (1996), "Contagious Currency Crises: First Tests", The Scandinavian Journal of Economics, Vol. 98, núm. 4, pp. 463-484.

Evangelist, M. y V. Sathe (2006), "Brazil’s 1998-1999 Currency Crisis”, documento de trabajo.

Feldstein, M. (2002), "Lessons from Argentina", Harvard University, Mimeo.

Forbes, K. y R. Rigobon (2001), "Measuring Contagion: Conceptual and Empirical Issues”, en: S. Claessens y K. Forbes (eds), International Financial Contagion, Kluwer Academic Publishers, Boston, pp. 43-66.

Forbes, K. y R. Rigobon (2002), "No Contagion, only Interdependence: Measuring Stock Market Co-Movements", The Journal of Finance, Vol. 57, núm. 5, pp. 2223-2261.

Forbes, K. (2004), "The Asian Flu and Russian Virus: The International Transmission of Crises in Firm-Level Data", Journal of International Economics, Vol. 63, núm. 1, pp. 59-92.

Frankel, J. y S. Schmukler (1998), "Crisis, Contagion, and Country Funds: Effects on East Asia and Latin America”, en: R. Glick (ed), Managing Capital Flows and Exchange Rates, Cambridge University Press, pp. 1-47.

Fondo Monetario Internacional (1999), Base de Datos de Estadísticas Financieras: recuperado de <www.imf.org > 
Fondo Monetario Internacional (2007), Base de Datos de Estadísticas Financieras: recuperado de <www.imf.org>.

Ganapolsky, E. y S. Schmukler (1998), "Crisis Management in Capital Markets: The Impact of Argentine Policy during the Tequila Effect", World Bank Policy Research, documento de trabajo, núm. 1951.

Geithner, T. (2003), "Lessons from the Crisis in Argentina", Fondo Monetario Internacional, Policy Development and Review Department, Octubre.

Gerlach, S. y F. Smets (1995), "Contagious Speculative Attacks”, European Journal of Political Economy, Vol. 11, núm.1, pp. 45-63.

Glick, R. y A. Rose (1999), "Contagion and Trade: Why are Currency Crises Regional?", Journal of International Money and Finance, Vol. 18, núm, 4, pp. 603-617.

Goldstein, M. (1998), “The Asian Financial Crisis: Causes, Cures, and Systemic Implications", Policy Analyses in International Economics, núm. 55.

Greene, W. (1997), Econometric Analysis, Prentice Hall, Tercera edición.

Hernández, L. y R. Valdés (2001), "What Drives Contagion: Trade, Neighborhood or Financial Links", International Review of Financial Analysis, Vol. 10, núm. 3, pp. 203-218.

IBGE (1998), Instituto Brasileiro de Geografía e Estadística, Clasificaciones Estadísticas, recuperado de <www.ibge.gov.br>.

INEGI (1998), Instituto Nacional de Estadística Geografía e Informática, Cuentas Nacionales, recuperado de <www.inegi.gob.mx>.

Jaque, F. (2004), "Emerging Market Economies: The Aftermath of Volatility Contagion in a Selection of Three Financial Crises", Banco Central de Chile, documento de trabajo, núm. 305.

Kaminsky, G. y S. Schmukler (1999), "What Triggers Market Jitters? A Chronical of the Asian Crisis", Journal of International Money and Finance, Vol. 18, núm.4, pp. 537-560.

Kaminsky, G., C. Reinhart y C. Végh (2003), “The Unholy Trinity of Financial Contagion", Journal of Economic Perspectives, Vol. 17, núm. 4, pp. 51-74.

Karolyi, A. (2003), "Does International Financial Contagion Really Exist?", Journal of International Finance, Vol. 6, núm. 2, pp. 179-199. 
EFECTOS DE LAS CRISIS ANTICIPADAS Y NO ANTICIPADAS SOBRE EL CONTAGIO FINANCIERO INTERNACIONAL

Kodres, L. y M. Pritsker (2002), "A Rational Expectations Model of Financial Contagion”, Journal of Finance, Vol. 57, núm. 2, pp. 769-799.

Kumar, M. y A. Peraud (2002), "Pure Contagion and Investors' Shifting Risk Appetite: Analytical Issues and Empirical Evidence", International Finance, Vol. 5, núm. 3, pp. 401-36.

Maletta, H. (2007), “A Catastrophe Foretold: Economic Reform, Crisis, Recovery, and Employment in Argentina", Universidad de El Salvador, Instituto de Investigación en Ciencias Sociales, documento de trabajo.

Moreno, R. (2002), "Learning from Argentina's Crisis", FRBSF Economic Letter, núm. 31, pp. 1-4.

Moser, T. (2003), "What is International Financial Contagion?", International Finance, Vol. 6, núm. 2, pp. 157-178.

Pritsker, M. (2001), “The Channels for Financial Contagion”, en: S. Claessens y K. Forbes (eds), International Financial Contagion, Kluwer Academic Publishers, Boston, pp. 67-98.

Perry, G. y L. Serven (2002), "The Anatomy and Physiology of a Multiple Crisis: Why was Argentina Special and what can we Learn from it", World Bank Policy Research, documento de trabajo, núm. 3081.

Radelet, S. y J. Sachs (1999), "What Have We Learned, So Far, From the Asian Financial Crisis", Harvard Institute for International Development, CAER Discussion Paper, núm. 37.

Reuters (2007), Información Histórica, recuperado de <www.reuters.com/finance>.

Reyes, A., "Desperate Measures", Asiaweek, 28 de agosto, 1999:50.

Sachs, J., A. Tornell, A. Velasco, G. Calvo y R. Cooper (1996), "Financial Crisis in Emerging Markets: The Lessons From 1995”, Brookings Papers On Economic Activity, Vol. 27, núm. 1, pp. 146-215.

Shleifer, A. y R. Vishny (1997), "The Limits of Arbitrage”, The Journal of Finance, Vol. 52, núm. 1, pp. 35-55.

Stiglitz, J. (1999), "Reforming the Global Economic Architecture: Lessons from Recent Crises". The Journal of Finance, núm. 54, pp. 1508-1521.

Valdés, R. (1997), "Emerging Markets Contagion: Evidence and Theory”, Banco Central de Chile, documento de trabajo, núm. 7. 
Van Rijckeghem, C. y B. Weder (2001), "Sources of Contagion: Is it Finance or Trade?”, Journal of International Economics, Vol. 54, núm. 2, pp. 293-308. Yam, J. (1999), "Hong Kong: From Crisis to Recovery", Hong Kong Monetary Authority, (Speech at HKMA Luncheon, London, Septiembre 28, 1999).

\section{ANEXO}

TABLA A1

Prueba LM de autocorrelación: Rendimientos accionarios y tasas de interés reales

\begin{tabular}{|l|l|l|l|l|}
\hline & \multicolumn{3}{|l|}{ Rendimiento de los índices accionarios } & Tasas de interés reales \\
\hline & Brasil & México & Brasil & México \\
\hline Hong Kong & $1426.5^{*}$ & $9844.67^{*}$ & $122.33^{*}$ & $67.14^{*}$ \\
\hline Argentina & $2093.74^{*}$ & $17770.47^{*}$ & $145.77^{*}$ & $90.35^{*}$ \\
\hline $\begin{array}{l}\text { * significativo al 1\% } \\
\text { Las pruebas se hacen luego de estimar la ecuación (1), para las dos variables dependientes de interés, con mínimos cuadrados } \\
\text { ordinarios. }\end{array}$
\end{tabular}

\section{TABLA A2}

Prueba White de heteroscedasticidad: Rendimientos accionarios y tasas de interés reales

\begin{tabular}{|l|l|l|l|l|}
\hline & \multicolumn{3}{|l|}{ Rendimiento índices accionarios } & \multicolumn{2}{l|}{ Tasas de interés reales } \\
\hline & Brasil & México & Brasil & México \\
\hline Hong Kong & $1670.17^{*}$ & $165.79^{*}$ & $70.31^{*}$ & $39.77^{*}$ \\
\hline Argentina & $100.95^{*}$ & $26.42^{*}$ & $14.34^{*}$ & $22.76^{*}$ \\
\hline $\begin{array}{l}\text { *significativo al 1\% } \\
\text { Las pruebas se hacen luego de estimar la ecuación (1), para las dos variables dependientes de interés, con mínimos cuadrados } \\
\text { ordinarios. }\end{array}$
\end{tabular}

